\title{
PAIR EXCITATIONS AND THE MEAN FIELD APPROXIMATION OF INTERACTING BOSONS, II
}

\author{
M. GRILLAKIS AND M. MACHEDON
}

\begin{abstract}
We consider a large number of Bosons with interaction potential $v_{N}(x)=N^{3 \beta} v\left(N^{\beta} x\right)$. In our earlier papers 21]-23] we considered a set of equations for the condensate $\phi$ and pair excitation function $k$ and proved that they provide a Fock space approximation to the exact evolution of a coherent state for $\beta<\frac{1}{3}$. In [24], in the hope of treating higher values of $\beta<1$, we introduced a coupled refinement of our original equations. In that paper, we showed the coupled equations conserve the number of particles and energy. In the current paper we prove that the coupled equations do indeed provide a Fock space approximation for $\beta<\frac{2}{3}$, at least locally in time. In order to do that, we re-formulate the equations of 24 in a way reminiscent of BBGKY and apply harmonic analysis techniques in the spirit of those used by X. Chen and J. Holmer in 10 to prove the necessary estimates. In turn, these estimates provide bounds for the pair excitation function $k$. While our earlier papers provide background material, the methods of this paper paper are mostly new, and the presentation is self-contained.
\end{abstract}

\section{INTRODUCTION}

The problem considered in this paper (as well as our earlier papers [21]- 24]) is the $N$-body linear Schrödinger equation

$$
\begin{aligned}
& \left(\frac{1}{i} \frac{\partial}{\partial t}-\sum_{j=1}^{N} \Delta_{x_{j}}+\frac{1}{N} \sum_{i<j} v_{N}\left(x_{i}-x_{j}\right)\right) \psi_{N}(t, \cdot)=0 \\
& \psi_{N}\left(0, x_{1}, \cdots, x_{N}\right) \sim \phi_{0}\left(x_{1}\right) \phi_{0}\left(x_{2}\right) \cdots \phi_{0}\left(x_{N}\right) \\
& \left\|\psi_{N}(t, \cdot)\right\|_{L^{2}\left(\mathbb{R}^{3 N}\right)}=1
\end{aligned}
$$

where $v_{N}(x):=N^{3 \beta} v\left(N^{\beta} x\right)$ with $0 \leq \beta \leq 1, v \in \mathcal{S}$ and $v \geq 0$. The meaning of $\sim$ in (2) will be made precise.

The goal is to find a rigorous, simple approximation (in a suitable norm) to $\psi_{N}$ which is consistent with

$$
\psi_{\text {approx }}\left(t, x_{1}, \cdots, x_{N}\right) \sim e^{i \chi(t)} \phi\left(t, x_{1}\right) \phi\left(t, x_{2}\right) \cdots \phi\left(t, x_{N}\right)
$$


as $N \rightarrow \infty$, where $\phi$ (which represents the Bose-Einstein condensate) satisfies a non-linear Schrödinger equation. The problem becomes more difficult, interesting, and requires new ideas as $\beta$ approaches 1 , and this explains why several authors, (including us) devoted several papers to their programs.

We refer to [37] for extensive background on (static) Bose-Einstein condensation.

During recent years, in a series of papers by Erdös and Yau [13], and Erdös, Schlein and Yau [14] to [16], it was proved

$$
\gamma_{1}^{N}\left(t, x, x^{\prime}\right) \rightarrow \bar{\phi}(t, x) \phi\left(t, x^{\prime}\right)
$$

in trace norm as $N \rightarrow \infty$, and similarly for the higher order marginal density matrices $\gamma_{k}^{N}$, where $k$ is fixed. Recent simplifications and generalizations were given in [31], [29], 6], [9], [10], [11]. See also [19], [28] for a different approach.

We also mention the approach based on the quantum de Finetti theorem in [7], as well as results in the case of negative interaction potentials in [12] and the different approach of Knowles and Pickl [28].

Another approach to this problem is based on Fock space techniques and the second quantization. In physics, it was pioneered in the papers by Bogoliubov [5], Lee, Huang and Yang [34] in the static case, and $\mathrm{Wu}[44$ in the time dependent case. See also the more recent papers 38, 39.

In the rigorous mathematical literature devoted to the time evolution problem, it originates in the of work Hepp [25], Ginibre and Velo [20] and, after lying dormant for about 30 years, Rodnianski and Schlein [41], followed by [21]. Currently, it is an active field.

Our project, initiated in collaboration with Margetis in [21], is to study a PDE describing additional second order corrections (given by a Bogoliubov transformation $e^{\mathcal{B}}$ ) to the right hand side of the approximation (3). Mathematically, Boguliubov transformations are representations of a group isomorphic to a real symplectic group, corresponding to the Segal-Shale-Weil representation in infinite dimensions, due to Shale. Interestingly, the theories seem to have evolved independently in physics and pure mathematics.

Several important recent papers also use coherent states and Bogoliubov transformations. These include [3] and [2]. In fact, Theorem 2.2 in [2] proves that the unitary operators of the type used in [25] and [20] can be obtained, abstractly, as Bogoliubov transformations. We 
mention in passing that our concrete 1 in $e^{\mathcal{B}}$ (to be discussed below) agrees with that Bogoliubov transformation (up to a phase), but only when applied to the vacuum. Roughly speaking, our $e^{\mathcal{B}}$ is not the operator corresponding to the evolution of the quadratic Bogoliubov Hamiltonian, but rather diagonalizes it.

In the current paper we initiate the analysis of solutions to a coupled system of PDEs for the condensate $\phi$ and pair excitation $k$, see(23), (24a $)-(24 b)$ below. It would be very interesting to obtain estimates up to the case $\beta<1$, as well as large time estimates for the solutions of these equations which are uniform in $N$, similar to those obtained in earlier works for the uncoupled equations (15a)-(15c) below) which describe the case $\beta<1 / 3$. We hope to address these question in future work. In this paper we only consider the case $1 / 3<\beta<2 / 3$, locally in time. As pointed out by one of our referees, it is unlikely that this type of construction, based on coherent states and Bogoliubov transformations, will work in the case $\beta=1$. This is because the construction is not sufficient to capture the ground state energy for the many body (static) case. The references for this phenomenon are [18], 45].

See also the paper by Lewin, Nam, and Schlein 35] for a Fock space type approach to an $L^{2}\left(\mathbb{R}^{3 N}\right)$ estimate based on corrections to a pure tensor product (Hartree state) rather than a coherent state. That approach has been generalized very recently to the case $\beta<1 / 3$ by Nam and Napiorkowski in [40, and their work uses the linear equations (15b), (15c) introduced in [23], as well as some of the estimates from that paper.

Very recently, after completing this work, we have also learned of the related paper [4]. A brief comparison of the results of that paper with ours is included at the end next section.

Also very recently we learned that, independently and in a different framework, Bach, Breteaux, Chen, Fröhlich and Sigal derived equations closely related to the equations of [24] and section (4) of our current paper in their recent work [1]. Those equations become equivalent to ours in the case of pure states.

\section{BACKGROUND AND STATEMENT OF THE MAIN RESUlT}

We start with a very brief review of symmetric Fock space. This is included for the convenience of the reader, and follows closely the exposition from our earlier papers. We refer the reader to [24] for more

\footnotetext{
${ }^{1}$ We mean that $\mathcal{B}=\mathcal{B}(k)$ where $k$ satisfies a PDE in $6+1$ dimensions and is, in principle, computable numerically .
} 
details and comments. The elements of $\mathcal{F}$ are vectors of the form

$$
\psi=\left(\psi_{0}, \psi_{1}\left(x_{1}\right), \psi_{2}\left(x_{1}, x_{2}\right), \ldots\right)
$$

where $\psi_{0} \in \mathbb{C}$ and $\psi_{k}$ are symmetric $L^{2}$ functions. The inner product is

$$
\langle\phi, \psi\rangle=\bar{\phi}_{0} \psi_{0}+\sum_{n=1}^{\infty} \int \bar{\phi}_{n} \psi_{n}
$$

Thus we use physicists' convention of an inner product linear in the second variable. The creation and annihilation distribution valued operators denoted by $a_{x}^{*}$ and $a_{x}$ respectively which act on vectors of the form $\left(0, \cdots, \psi_{n-1}, 0, \cdots\right)$ and $\left(0, \cdots, \psi_{n+1}, 0, \cdots\right)$ by

$$
\begin{aligned}
& a_{x}^{*}\left(\psi_{n-1}\right):=\frac{1}{\sqrt{n}} \sum_{j=1}^{n} \delta\left(x-x_{j}\right) \psi_{n-1}\left(x_{1}, \ldots, x_{j-1}, x_{j+1}, \ldots, x_{n}\right) \\
& a_{x}\left(\psi_{n+1}\right):=\sqrt{n+1} \psi_{n+1}\left([x], x_{1}, \ldots, x_{n}\right)
\end{aligned}
$$

with $[x]$ indicating that the variable $x$ is frozen. The vacuum state is defined as follows:

$$
\Omega:=(1,0,0 \ldots)
$$

One can easily check the canonical relations $\left[a_{x}, a_{y}^{*}\right]=\delta(x-y)$ and since the creation and annihilation operators are distribution valued we can form operators that act on $\mathcal{F}$ by introducing a field, say $\phi(x)$, and form

$$
a(\bar{\phi}):=\int d x\left\{\bar{\phi}(x) a_{x}\right\} \quad \text { and } \quad a^{*}(\phi):=\int d x\left\{\phi(x) a_{x}^{*}\right\}
$$

where by convention we associate $a$ with $\bar{\phi}$ and $a^{*}$ with $\phi$. Also define the skew-Hermitian operator

$$
\mathcal{A}(\phi):=\int d x\left\{\bar{\phi}(x) a_{x}-\phi(x) a_{x}^{*}\right\}
$$

and coherent states

$$
\psi:=e^{-\sqrt{N} \mathcal{A}(\phi)} \Omega
$$

It is easy to check that

$e^{-\sqrt{N} \mathcal{A}(\phi)} \Omega=\left(\ldots c_{n} \prod_{j=1}^{n} \phi\left(x_{j}\right) \ldots\right) \quad$ with $\quad c_{n}=\left(e^{\left.-N\|\phi\|_{L^{2}}^{2} N^{n} / n !\right)^{1 / 2}}\right.$.

We also consider

$$
\mathcal{B}(k):=\frac{1}{2} \int d x d y\left\{\bar{k}(t, x, y) a_{x} a_{y}-k(t, x, y) a_{x}^{*} a_{y}^{*}\right\} .
$$

This particular construction and the corresponding unitary operator $\mathcal{M}:=e^{-\sqrt{N} \mathcal{A}} e^{-\mathcal{B}}$ were introduced (at least in the mathematics 
literature related to the problem under consideration) in [21]. The construction is in the spirit of Bogoliubov theory in physics, and the Segal-Shale-Weil representation in mathematics.

The Fock Hamiltonian is

$$
\begin{aligned}
\mathcal{H} & :=\mathcal{H}_{1}-\frac{1}{N} \mathcal{V} \quad \text { where, } \\
\mathcal{H}_{1} & :=\int d x d y\left\{\Delta_{x} \delta(x-y) a_{x}^{*} a_{y}\right\} \quad \text { and } \\
\mathcal{V} & :=\frac{1}{2} \int d x d y\left\{v_{N}(x-y) a_{x}^{*} a_{y}^{*} a_{x} a_{x}\right\},
\end{aligned}
$$

where $v_{N}(x)=N^{3 \beta} v\left(N^{\beta} x\right)$. It is a diagonal operator on Fock space, and it acts as a differential operator in $n$ variable

$$
H_{n, P D E}=\sum_{j=1}^{n} \Delta_{x_{j}}-\frac{1}{N} \sum_{i<j} N^{3 \beta} v\left(N^{\beta}\left(x_{j}-x_{k}\right)\right)
$$

on the $n$th component of $\mathcal{F}$. Notice this is the same as (1), except that the dimension $n$ is decoupled from the parameter $N$.

Our goal is to study the evolution of (possibly modified) coherent initial conditions of the form

$$
\psi_{\text {exact }}=e^{i t \mathcal{H}} e^{-\sqrt{N} \mathcal{A}\left(\phi_{0}\right)} e^{-\mathcal{B}\left(k_{0}\right)} \Omega
$$

In our earlier papers [21, 22, 23, 24] we considered an approximation of the form

$$
\psi_{\text {appr }}:=e^{-\sqrt{N} \mathcal{A}(\phi(t)} e^{-\mathcal{B}(k(t))} \Omega
$$

and derived suitable Schrödinger type equations equations for $\phi(t, x)$, $k(t, x, y)$ so that $\psi_{\text {exact }}(t) \approx e^{i N \chi(t)} \psi_{\text {appr }}(t)$, with $\chi(t)$ a real phase factor, in order to find precise estimates in Fock space, see Theorem (2.1) below. Our strategy is to consider

$$
\psi_{\text {red }}=e^{\mathcal{B}(t)} e^{\sqrt{N} \mathcal{A}(t)} e^{i t \mathcal{H}} e^{-\sqrt{N} \mathcal{A}(0)} e^{-\mathcal{B}(0)} \Omega
$$

and then compute a "reduced Hamiltonian"

$$
H_{\text {red }}=\frac{1}{i}\left(\partial_{t} \mathcal{M}^{*}\right) \mathcal{M}+\mathcal{M}^{*} \mathcal{H} \mathcal{M}
$$

so that

$$
\frac{1}{i} \partial_{t} \psi_{\text {red }}=\mathcal{H}_{\text {red }} \psi_{\text {red }} \text {. }
$$

To state the results of [21, 22, 23] we define the operator kernel

$$
\begin{aligned}
g_{N}(t, x, y) & :=-\Delta_{x} \delta(x-y)+\left(v_{N} *|\phi|^{2}\right)(t, x) \delta(x-y) \\
& +v_{N}(x-y) \bar{\phi}(t, x) \phi(t, y)
\end{aligned}
$$


and

$$
\begin{aligned}
& \mathbf{S}_{\text {old }}(s):=\frac{1}{i} s_{t}+g_{N}^{T} \circ s+s \circ g_{N} \text { and } \\
& \mathbf{W}_{\text {old }}(p):=\frac{1}{i} p_{t}+\left[g_{N}^{T}, p\right] \\
& m_{N}(x, y):=-v_{N}(x-y) \phi(x) \phi(y), v_{N}(x)=N^{3 \beta} v\left(N^{\beta} x\right)
\end{aligned}
$$

The main result of [23] can be summarized as follows:

Theorem 2.1. Let $\phi$ and $k$ satisfy

$$
\begin{aligned}
& \frac{1}{i} \partial_{t} \phi-\Delta \phi+\left(v_{N} *|\phi|^{2}\right) \phi=0 \\
& \mathbf{S}_{\text {old }}(\operatorname{sh}(2 k))=m_{N} \circ \operatorname{ch}(2 k)+\overline{\operatorname{ch}(2 k)} \circ m_{N} \\
& \mathbf{W}_{\text {old }}(\overline{\operatorname{ch}(2 k)})=m_{N} \circ \overline{\operatorname{sh}(2 k)}-\operatorname{sh}(2 k) \circ \bar{m}_{N} .
\end{aligned}
$$

with prescribed initial conditions $\phi(0, \cdot)=\phi_{0}, k(0, \cdot, \cdot)=0$. If $\phi, k$ satisfy the above equations, then there exists a real phase function $\chi$ such that

$$
\left\|\psi_{\text {exact }}(t)-e^{i N \chi(t)} \psi_{\text {appr }}(t)\right\|_{\mathcal{F}} \leq \frac{C(1+t) \log ^{4}(1+t)}{N^{(1-3 \beta) / 2}} .
$$

provided $0<\beta<\frac{1}{3}$.

See [23] for the reasons behind these equations. We also mention a very recent simple derivation of these equations in [40]. This result was extended to the case $\beta<\frac{1}{2}$ in 33 , where it was also argued informally that the equations of [23] do not provide an approximation for $\beta>\frac{1}{2}$.

In the hope of obtaining an approximation for higher $\beta$, in [24] we introduced a coupled refinement of the system (15a), (15b), (15c).

The coupled equations of [24] were introduced the following way: Since $\mathcal{H}_{\text {red }}$ is a fourth order polynomial in $a$ and $a^{*}$,

$$
\mathcal{H}_{\text {red }} \Omega=\left(X_{0}, X_{1}, X_{2}, X_{3}, X_{4}, 0, \cdots\right) .
$$

The new, coupled equations for $\phi$ and $k$ that we introduce in [24] can be written abstractly as

$$
X_{1}=0 \text { and } X_{2}=0 \text {. }
$$

It was shown there that they are Euler-Lagrange equations for the Lagrangian density $X_{0}$, and that their solutions preserve the number of particles and the energy. See also [1].

Remark 2.2. The static terms of $X_{0}(t)$ (not involving time derivatives) also appear in the recent paper [3], but do not serve as a Lagrangian there. 
To write down the equations (18) explicitly in terms of $\phi$ and $k$, we introduce

Definition 2.3. Define

$$
\begin{aligned}
& \Lambda\left(t, x_{1}, x_{2}\right)=\frac{1}{2 N} \operatorname{sh}(2 k)\left(t, x_{1}, x_{2}\right)+\phi\left(t, x_{1}\right) \phi\left(t, x_{2}\right) \\
& \Gamma\left(t, x_{1}, x_{2}\right)=\frac{1}{N}(\overline{\operatorname{sh}(k)} \circ \operatorname{sh}(k))\left(t, x_{1}, x_{2}\right)+\bar{\phi}\left(t, x_{1}\right) \phi\left(t, x_{2}\right)
\end{aligned}
$$

and the new operator kernel

$$
\begin{aligned}
& \tilde{g}_{N}(t, x, y):=-\Delta_{x} \delta(x-y)+v_{N}(x-y)(\operatorname{tr} \Gamma)(t, x) \delta(x-y) \\
& +v_{N}(x-y) \Gamma(t, x, y)
\end{aligned}
$$

where $\operatorname{tr}$ denotes trace density, and define

$$
\tilde{\mathbf{S}}(s):=\frac{1}{i} s_{t}+\tilde{g}_{N}^{T} \circ s+s \circ \tilde{g}_{N} \quad \text { and } \quad \tilde{\mathbf{W}}(p):=\frac{1}{i} p_{t}+\left[\tilde{g}_{N}^{T}, p\right]
$$

In this notation, the following is proved in [24]

Theorem 2.4. The equation $X_{1}=0$ is equivalent to

$$
\begin{aligned}
& \frac{1}{i} \partial_{t} \phi(t, x)-\Delta \phi+\int v_{N}(x-y) \Lambda(t, x, y) \bar{\phi}(t, y) d y \\
& +\frac{1}{N}\left(v_{N} * \operatorname{Tr}(\operatorname{sh}(k) \circ \overline{\operatorname{sh}(k)})\right)(t, x) \phi(t, x) \\
& +\frac{1}{N} \int v_{N}(x-y)(\operatorname{sh}(k) \circ \overline{\operatorname{sh}(k)})(t, x, y) \phi(t, y) d y=0
\end{aligned}
$$

Here $\operatorname{Tr}(\operatorname{sh}(k) \circ \overline{\operatorname{sh}(k)})(t, x)=(\operatorname{sh}(k) \circ \overline{\operatorname{sh}(k)})(t, x, x)$ denotes the trace density.

The equation $X_{2}=0$ is equivalent to

$$
\begin{array}{r}
\tilde{\mathbf{S}}(\operatorname{sh}(2 k))+\left(v_{N} \Lambda\right) \circ \operatorname{ch}(2 k)+\overline{\operatorname{ch}(2 k)} \circ\left(v_{N} \Lambda\right)=0 \\
\tilde{\mathbf{W}}(\overline{\operatorname{ch}(2 k)})+\left(v_{N} \Lambda\right) \circ \overline{\operatorname{sh}(2 k)}-\operatorname{sh}(2 k) \circ\left(\overline{v_{N} \Lambda}\right)=0
\end{array}
$$

Notice the similarity with (15a), (15b), (15c).

Since it is difficult to prove estimates for these equations directly, we will write them down in a different, equivalent form. The derivation will be self-contained, and in fact most of the rest of this paper is independent of our previous work [21]-[24].

We now state the main result of our current paper.

Theorem 2.5. Let $\frac{1}{3}<\beta<\frac{2}{3}$, and let the interaction potential $v \in \mathcal{S}$ satisfy $v \geq 0$ and $|\hat{v}| \leq \hat{w}$ for some $w \in \mathcal{S}$. Let $\phi, k$ be solutions to (23), (24a), (24b) with smooth initial conditions $\phi(0, \cdot), k(0, \cdot)$ satisfying the following regularity uniformly in $N$ (expressed in terms of $\phi, \Lambda$ and $\Gamma$ 
defined above, as well as $\operatorname{sh}(k))$ : For some $\epsilon_{0}>0$ and all $0 \leq i \leq 1$, $0 \leq j \leq 2$

$$
\begin{aligned}
& \left\|<\nabla_{x}>\left.^{\frac{1}{2}+\epsilon_{0}}\left(\frac{\partial}{\partial t}\right)^{i} \nabla_{x}^{j} \phi(t, x)\right|_{t=0}\right\|_{L^{2}(d x)} \leq C \\
& \left\|<\nabla_{x}>^{\frac{1}{2}+\epsilon_{0}}<\nabla_{y}>\left.^{\frac{1}{2}+\epsilon_{0}}\left(\frac{\partial}{\partial t}\right)^{i} \nabla_{x+y}^{j} \Gamma(t, x, y)\right|_{t=0}\right\|_{L^{2}(d x d y)} \leq C \\
& \left\|<\nabla_{x}>^{\frac{1}{2}+\epsilon_{0}}<\nabla_{y}>\left.^{\frac{1}{2}+\epsilon_{0}}\left(\frac{\partial}{\partial t}\right)^{i} \nabla_{x+y}^{j} \Lambda(t, x, y)\right|_{t=0}\right\|_{L^{2}(d x d y)} \leq C \\
& \left\|\nabla_{x+y}^{j} \operatorname{sh}(k)(0, x, y)\right\|_{L^{2}(d x d y)} \leq C
\end{aligned}
$$

Then there exists a real function $\chi(t)=\chi_{N}(t)$ and a (small) $T_{0}>0$ and $C=C\left(T_{0}, \epsilon_{0}, \beta\right)$ such that

$$
\begin{aligned}
& \left\|\psi_{\text {exact }}-\psi_{\text {appr }}\right\|_{\mathcal{F}}:=\left\|e^{i t \mathcal{H}} e^{-\sqrt{N} \mathcal{A}\left(\phi_{0}\right)} e^{-\mathcal{B}(k(0))} \Omega-e^{i \chi(t)} e^{-\sqrt{N} \mathcal{A}(\phi(t))} e^{-\mathcal{B}(k(t))} \Omega\right\|_{\mathcal{F}} \\
& \leq \frac{C}{N^{\frac{1}{6}}} \\
& \text { for } 0 \leq t \leq T_{0} .
\end{aligned}
$$

Several remarks are in order.

Remark 2.6. First, we comment on the initial conditions for our equations. The kernel considered in [3] (for $\beta=1$ ) is of the form $k(t, x, y)=$ $-N \phi(t, x) \phi(t, y) w(N(x-y))$ where $\phi$ solves the Gross-Pitaevskii NLS and $1-w$ is the solution to the zero energy scattering solution. The function $w(x)$ is smooth near 0 and behaves like $\frac{a}{|x|}$ at infinity. This is close to the expected form of the ground state and corresponds, in our set-up, to

$$
\Lambda(0, x, y)=\phi(x) \phi(y)\left(1-N^{\beta-1} w\left(N^{\beta}(x-y)\right)\right.
$$

We can prescribe $\Lambda(0, x, y)$ arbitrarily, but the time derivative is determined by the equation (33b) (see Section (4)) which has a singular term $\frac{1}{N} v_{N}$ and

$\frac{1}{i} \partial_{t} \Lambda(t, x, y)=-\left(-\Delta_{x}-\Delta_{y}+\frac{1}{N} v_{N}(x-y)\right) \Lambda(t, x, y)+$ smoother terms

Our initial conditions (27) with one time derivative are compatible with such initial condition provided

$$
\begin{aligned}
& <\nabla_{x}>^{\frac{1}{2}+\epsilon_{0}}<\nabla_{y}>^{\frac{1}{2}+\epsilon_{0}}\left(-\Delta_{x, y}+\frac{1}{N} v_{N}(x-y)\right) \phi(x) \phi(y)\left(1-N^{\beta-1} w\left(N^{\beta}(x-y)\right)\right. \\
& \in L^{2}(d x d y)
\end{aligned}
$$


which is true if, for instance,

$$
\left(-\Delta_{x, y}+\frac{1}{N} v_{N}(x-y)\right)\left(1-N^{\beta-1} w\left(N^{\beta}(x-y)\right)=0\right.
$$

but are incompatible with the choice $k=0, \Lambda(0, x, y)=\phi(x) \phi(y)$. This is in contrast to our earlier results [21, 22, 23] on the uncoupled equations.

Remark 2.7. The value $\beta<2 / 3$ is the highest value for which the potential $N^{3 \beta-1} v\left(N^{\beta}(x-y)\right)$ can be treated as a perturbation using Strichartz estimates in various places in the paper (see the next section). We hope it will be possible to develop a more refined analysis which does not treat the potential as a perturbation, and extend the range of $\beta$.

Remark 2.8. Our theorem is stated and proved only locally in (small) time. The proof is based on Theorem (6.1) which is, essentially, a local existence theorem with bounds uniform in $N$ for initial conditions with

$$
\begin{aligned}
& \left\|<\nabla_{x}>^{\frac{1}{2}+\epsilon}<\nabla_{y}>^{\frac{1}{2}+\epsilon} \Lambda(0, \cdot)\right\|_{L^{2}} \leq C \\
& \left\|<\nabla_{x}>^{\frac{1}{2}+\epsilon}<\nabla_{y}>^{\frac{1}{2}+\epsilon} \Gamma(0, \cdot)\right\|_{L^{2}} \leq C \\
& \left\|<\nabla_{x}>^{\frac{1}{2}+\epsilon} \phi(0, \cdot)\right\|_{L^{2}} \leq C
\end{aligned}
$$

However, one can show, based on the conservation laws proved in [24], for $\epsilon$ sufficiently small,

$$
\begin{aligned}
& \left\|<\nabla_{x}>^{\frac{1}{2}+\epsilon}<\nabla_{y}>^{\frac{1}{2}+\epsilon} \Lambda(t, \cdot)\right\|_{L^{2}} \leq C(t) \\
& \left\|<\nabla_{x}>^{\frac{1}{2}+\epsilon}<\nabla_{y}>^{\frac{1}{2}+\epsilon} \Gamma(t, \cdot)\right\|_{L^{2}} \leq C \\
& \left\|<\nabla_{x}>^{\frac{1}{2}+\epsilon} \phi(t, \cdot)\right\|_{L^{2}} \leq C
\end{aligned}
$$

uniformly in $N$. This makes it likely that our main result Theorem (2.5) extends globally in time with some constant $C=C(t)$ depending on $t$. We do not yet know how $C(t)$ will depend on $t$ as $t \rightarrow \infty$.

Remark 2.9. In the very recent and important paper [4], Bocatto, Cenatiempo and Schlein prove a result closely related to ours in the full range $\beta<1$, and the estimate is global in time. However, there are substantial differences between their work and ours. The techniques used in [4] are quite different than ours, and the approximation in [4] is given (translating to our notation) by $e^{i \chi(t)} e^{-\sqrt{N} \mathcal{A}(\phi(t))} e^{-\mathcal{B}(k(t))} U_{2, N}(t) \Omega$ where $k(t)=k(t, x, y)$ is explicit (and related but different from our $k(t)$ ) and $U_{2, N}(t)$ is an evolution in Fock space with a quadratic generator (see the page preceding Theorem 1.1 in [4]). Given the complexity the evolution equation defining of $U_{2, N}(t)$, we believe there is still sufficient interest in 
having an approximation given by just $e^{i \chi(t)} e^{-\sqrt{N} \mathcal{A}(\phi(t))} e^{-\mathcal{B}(k(t))} \Omega$ where $k$ satisfies a classical PDE in $6+1$ variables.

\section{Guide to The PROOF}

Before going into the details of the complete proof of the Main Theorem (2.5), we will explain the main ideas. In order to control the error terms in section (9), we need estimates for $\|\operatorname{sh}(2 k)\|_{L^{2}(d x d y)}$, uniformly in $N$. This is accomplished in Section (7), but here is a miniature simplified sketch. ( $\mathbf{S}$ denotes $\frac{1}{i} \frac{\partial}{\partial t}-\Delta$ in $3+1$ or $6+1$ dimensions.)

Replace equation (24a) by the simplified version

$$
\mathbf{S} u=-v_{N}(x-y) \Lambda(t, x, y) \sim-\delta(x-y) \Lambda(t, x, x)
$$

( $u$ denotes $\operatorname{sh}(2 k)$ ). The right hand side is too singular to apply energy estimates or Strichartz estimates, so we proceed by writing Duhamel's formula and integrating by parts:

$$
\begin{aligned}
& u(t, x, y)=u(0, x, y)+\int_{0}^{t} e^{i(t-s) \Delta_{x, y}} \delta(x-y) \Lambda(s, x, x) d s \\
& =\int_{0}^{t} e^{i(t-s) \Delta_{x, y}} \Delta_{x, y}^{-1} \frac{\partial}{\partial s}(\delta(x-y) \Lambda(s, x, x)) d s \\
& + \text { boundary terms }
\end{aligned}
$$

Now $\Delta_{x, y}^{-1}$ smoothes out the singularity of $\delta(x-y)$, but we need good estimates for

$$
\frac{\partial}{\partial s} \Lambda(s, x, x)
$$

Therefore, we need to assume good estimates for $\left.\frac{\partial}{\partial s} \Lambda(s, x, y)\right|_{s=0}$. Continuing, we derive coupled equations for $\phi, \Lambda$ and $\Gamma$, see Theorem (4.1). For this discussion, just look at the simplified model:

$$
\begin{aligned}
\mathbf{S} \phi(t, x) & =-\int d z v_{N}(x-z) \Lambda(t, x, z) \bar{\phi}(t, x) \\
\left(\mathbf{S}+\frac{1}{N} v_{N}\right) \Lambda(t, x, y)= & -\int d z v_{N}(x-z) \Lambda(t, x, z) \bar{\phi}(t, z) \phi(t, y) \\
& -\int d z v_{N}(y-z) \Lambda(t, y, z) \bar{\phi}(t, z) \phi(t, x)
\end{aligned}
$$


or, replacing $v_{N}$ by $\delta$,

$$
\begin{aligned}
\mathbf{S} \phi(t, x) & =-\Lambda(t, x, x) \bar{\phi}(t, x) \\
\left(\mathbf{S}+\frac{1}{N} v_{N}(x-y)\right) \Lambda(t, x, y)= & -\Lambda(t, x, x) \bar{\phi}(t, x) \phi(t, y) \\
& -\Lambda(t, y, y) \bar{\phi}(t, y) \phi(t, x)
\end{aligned}
$$

It is well known that NLS is well-posed in $H^{1 / 2}$ in $3+1$ dimensions, so it is natural to prove a well-posedness result with $\nabla^{1 / 2} \phi(0, x) \in L^{2}$ and $\nabla_{x}^{1 / 2} \nabla_{y}^{1 / 2} \Lambda(0, x, y) \in L^{2}$. To get things started, we need the space-time collapsing estimate (53) of Lemma (5.1). This holds for solutions of the homogeneous Schrödinger equations, and, automatically, in $X^{1 / 2+}$ spaces (see section (5) for the definition and properties of these spaces). Ignoring the potential for a moment, it is very easy to treat the equation (32).

$$
\begin{aligned}
\mathbf{S}\left(\nabla_{x}^{1 / 2} \nabla_{y}^{1 / 2} \Lambda(t, x, y)\right)= & -\nabla_{x}^{1 / 2}(\Lambda(t, x, x) \bar{\phi}(t, x)) \nabla_{y}^{1 / 2} \phi(t, y) \\
& -\nabla_{y}^{1 / 2}(\Lambda(t, y, y) \bar{\phi}(t, y)) \nabla_{x}^{1 / 2} \phi(t, x)
\end{aligned}
$$

If $\nabla^{1 / 2} \phi \in X^{1 / 2+}$ and $\nabla_{x}^{1 / 2} \nabla_{y}^{1 / 2} \Lambda \in X^{1 / 2+}$ then, locally in time, $\nabla^{1 / 2} \phi \in L^{\infty}(d t) L^{2}(d x)$ and $\nabla_{x}^{1 / 2} \Lambda(t, x, x) \in L^{2}(d t) L^{2}(d x)$ by (53), and thus

$$
\begin{aligned}
\nabla_{x}^{1 / 2}(\Lambda(t, x, x) \bar{\phi}(t, x)) \nabla_{y}^{1 / 2} \phi(t, y) & \in L^{2}(d t) L^{6 / 5}(d x) L^{2}(d y) \\
& \subset X^{-1 / 2-}
\end{aligned}
$$

and similarly for the second term, which puts $\nabla_{x}^{1 / 2} \nabla_{y}^{1 / 2} \Lambda(t, x, y)$ back in $X^{1 / 2+}$ (once we resolve the technical discrepancy between $1 / 2+$ and $1 / 2$ - by introducing some epsilons), closing the loop in $X$ spaces. The argument for equation (31) is similar.

When applying these estimates to (32) we also have to differentiate the potential term, and $\nabla_{x}^{1 / 2} \nabla_{y}^{1 / 2} \frac{1}{N} v_{N} \in L^{6 / 5}$ uniformly in $N$ if $\beta \leq$ $2 / 3$. This is the lowest exponent for which the term can be treated as a perturbation using $L^{2}(d t) L^{6 / 5}(d(x-y)) L^{2}(d(x+y))$ Strichartz estimates. The precise statement is Proposition (5.7).

The spaces $X^{1 / 2+}$ come with a fixed cut-off function, and we need to vary the size of the time interval at will in order to obtain a contraction out of the argument outlined above, so, for this technical reason, the non-linear result Theorem (6.1) is proved in the spaces (72)-(74). Once

\footnotetext{
$21 / 2+$ is a number slightly bigger than $1 / 2$.
} 
we have this result, we can differentiate the equations with respect to $t$ and get control over $\mathbf{N}_{T}\left(\frac{\partial}{\partial t} \Lambda\right)$ which in turn controls (30). Finally, in order to control Fock space error terms such as

$$
\begin{aligned}
& \left(\mathbf{S}+\frac{1}{N} \sum_{1 \leq i<j \leq 3} v_{N}\left(x_{i}-x_{j}\right)\right) E=\frac{1}{\sqrt{N}} \phi\left(x_{1}\right) v_{N}\left(x_{1}-x_{2}\right) \operatorname{sh}(k)\left(x_{2}, x_{3}\right) \\
& E(0, \cdot)=0
\end{aligned}
$$

(see (92) ) we refrain from using time derivatives of $\operatorname{sh}(k)$ which would require higher derivatives of $\Lambda$ (see (29)). Instead we use Strichartz estimates. The fact that $\frac{1}{\sqrt{N}}\left\|v_{N}\right\|_{L^{6 / 5}} \leq \frac{C}{N^{1 / 6}}($ for $\left.\beta \leq 2 / 3)\right)$ explains the exponent in the statement of the theorem. The complete proof has to include several iterates of this type of argument together with energy estimates in Fock space in order to handle off diagonal terms in our reduced Hamiltonian.

\section{The Equations For $\Lambda$ AND $\Gamma$ (SElf-CONTAined DERIVATiOn)}

As already mentioned, it seems difficult to obtain estimates (uniformly in $N$ ) for $\phi$ and $k$ directly from equations (24a), (24b). The equations seem linear, but the "coefficients" $v_{N} \Lambda, v_{N} \Gamma$ depend on $\operatorname{sh}(2 k), \operatorname{ch}(2 k)$. We will proceed indirectly by deriving and studying equations for $\Lambda$ and $\Gamma$. 
Theorem 4.1. The equations of Theorem (2.4) are equivalent to

$$
\begin{aligned}
& \left\{\frac{1}{i} \partial_{t}-\Delta_{x_{1}}\right\} \phi\left(x_{1}\right)=-\int d y\left\{v_{N}\left(x_{1}-y\right) \Gamma(y, y)\right\} \phi\left(x_{1}\right) \\
- & \int d y\left\{v_{N}\left(x_{1}-y\right) \phi(y)\left(\Gamma\left(y, x_{1}\right)-\bar{\phi}(y) \phi\left(x_{1}\right)\right)+v_{N}\left(x_{1}-y\right) \bar{\phi}(y)\left(\Lambda\left(x_{1}, y\right)-\phi\left(x_{1}\right) \phi(y)\right)\right\} \\
& \left\{\frac{1}{i} \partial_{t}-\Delta_{x_{1}}-\Delta_{x_{2}}+\frac{1}{N} v_{N}\left(x_{1}-x_{2}\right)\right\} \Lambda\left(x_{1}, x_{2}\right) \\
= & -\int d y\left\{v_{N}\left(x_{1}-y\right) \Gamma(y, y)+v_{N}\left(x_{2}-y\right) \Gamma(y, y)\right\} \Lambda\left(x_{1}, x_{2}\right) \\
- & \int d y\left\{\left(v_{N}\left(x_{1}-y\right)+v_{N}\left(x_{2}-y\right)\right)\left(\Lambda\left(x_{1}, y\right) \Gamma\left(y, x_{2}\right)+\bar{\Gamma}\left(x_{1}, y\right) \Lambda\left(y, x_{2}\right)\right)\right\}+ \\
+ & 2 \int d y\left\{\left(v_{N}\left(x_{1}-y\right)+v_{N}\left(x_{2}-y\right)\right)|\phi(y)|^{2} \phi\left(x_{1}\right) \phi\left(x_{2}\right)\right\} \\
& \left\{\frac{1}{i} \partial_{t}-\Delta_{x_{1}}+\Delta_{x_{2}}\right\} \bar{\Gamma}\left(x_{1}, x_{2}\right) \\
= & -\int d y\left\{\left(v_{N}\left(x_{1}-y\right)-v_{N}\left(x_{2}-y\right)\right) \Lambda\left(x_{1}, y\right) \bar{\Lambda}\left(y, x_{2}\right)\right\}+ \\
- & \int d y\left\{\left(v_{N}\left(x_{1}-y\right)-v_{N}\left(x_{2}-y\right)\right)\left(\bar{\Gamma}\left(x_{1}, y\right) \bar{\Gamma}\left(y, x_{2}\right)+\bar{\Gamma}(y, y) \bar{\Gamma}\left(x_{1}, x_{2}\right)\right)\right\} \\
+ & 2 \int d y\left\{\left(v_{N}\left(x_{1}-y\right)-v_{N}\left(x_{2}-y\right)\right)|\phi(y)|^{2} \phi\left(x_{1}\right) \bar{\phi}\left(x_{2}\right)\right\}
\end{aligned}
$$

See (40a)-(40c) for the conceptual meaning of these equations in terms of the density matrices $\mathcal{L}$ defined below. Also note that $\phi, \Lambda$ and $\Gamma$ depend on $t$, but this dependence has been suppressed in the above formulas.

While it is easy to prove this by direct calculation, we proceed with a derivation which is independent of Theorem (2.4) of our previous paper [24].

As in our previous papers [21]-[24], we consider

$$
\begin{array}{rlrl}
\mathcal{M} & :=e^{-\sqrt{N} \mathcal{A}} e^{-\mathcal{B}} & & \text { and we have the evolution } \\
\frac{1}{i} \partial_{t} \mathcal{M} & =\mathcal{H} \mathcal{M}-\mathcal{M} \mathcal{H}_{\text {red }} & \text { and of course, } \\
-\frac{1}{i} \partial_{t} \mathcal{M}^{*} & =\mathcal{M}^{*} \mathcal{H}-\mathcal{H}_{\text {red }} \mathcal{M}^{*} . &
\end{array}
$$

The evolution equation for $\mathcal{M}$ above is obvious from (11). 
Take a monomial of the form: (Wick ordered)

$$
\mathcal{P}_{m, n}=a_{y_{1}}^{*} a_{y_{2}}^{*} \ldots a_{y_{m}}^{*} a_{x_{1}} a_{x_{2}} \ldots a_{x_{n}}
$$

and define the $\mathcal{L}$ matrices as follows,

$$
\begin{aligned}
& \mathcal{L}_{m, n}\left(t, y_{1}, \ldots, y_{m} ; x_{1}, \ldots, x_{n}\right):=\frac{1}{N^{(m+n) / 2}}\left\langle a_{y_{1}} \cdots a_{y_{m}} \mathcal{M} \Omega, a_{x_{1}} \cdots a_{x_{n}} \mathcal{M} \Omega\right\rangle \\
& =\frac{1}{N^{(m+n) / 2}}\left\langle\Omega, \mathcal{M}^{*} \mathcal{P}_{m, n} \mathcal{M} \Omega\right\rangle
\end{aligned}
$$

The notation is chosen so that the second set of variables are un-barred. We will often skip the $t$ dependence, since it is passive in the calculations that we have in mind.

Fortunately we will only need $\mathcal{L}_{0,1}, \mathcal{L}_{1,1}$ and $\mathcal{L}_{0,2}$ (which turn out to be $\phi, \Gamma$ and $\Lambda$ ) but the computation is quite general.

To get started, we observe that from the evolution of the operator $\mathcal{M}$ we have,

$$
\begin{aligned}
& \frac{1}{i} \partial_{t}\left(\mathcal{M}^{*} \mathcal{P} \mathcal{M}\right)=\left[\mathcal{H}_{\text {red }}, \mathcal{M}^{*} \mathcal{P} \mathcal{M}\right]+\mathcal{M}^{*}[\mathcal{P}, \mathcal{H}] \mathcal{M} \\
& \frac{1}{i} \partial_{t} \mathcal{L}=\frac{1}{N^{(n+m) / 2}}\left(\left\langle\Omega,\left[\mathcal{H}_{\mathrm{red}}, \mathcal{M}^{*} \mathcal{P} \mathcal{M}\right] \Omega\right\rangle+\left\langle\Omega, \mathcal{M}^{*}[\mathcal{P}, \mathcal{H}] \mathcal{M} \Omega\right\rangle\right) .
\end{aligned}
$$

At this point we record the following lemma

Lemma 4.2. If $\mathcal{H}_{\mathrm{red}} \Omega=\left(\mu, 0,0, X_{3}, X_{4}, 0 \ldots\right)$ and

$$
\mathcal{P}:=a \quad \text { or } \quad \mathcal{P}:=a a \quad \text { or } \quad \mathcal{P}:=a^{*} a
$$

then

$$
\left\langle\Omega,\left[\mathcal{H}_{\mathrm{red}}, \mathcal{M}^{*} \mathcal{P} \mathcal{M}\right] \Omega\right\rangle=0,
$$

leaving only the second term in (37)

Proof. We use the following notation:

$$
\begin{aligned}
& c=\operatorname{ch}(k), u=\operatorname{sh}(k) \\
& a_{x}(c):=\int d y\left\{a_{y} c(y, x)\right\}, \quad a_{x}^{*}(u):=\int d y\left\{a_{y}^{*} u(y, x)\right\} \\
& a_{x}^{*}(\bar{c}):=\int d y\left\{a_{y}^{*} \bar{c}(y, x)\right\}=\int d y\left\{c(x, y) a_{y}^{*}\right\} \quad \text { by symmetry } \\
& a_{x}(\bar{u}):=\int d y\left\{a_{y} \bar{u}(y, x)\right\}=\int d y\left\{\bar{u}(x, y) a_{y}\right\} \quad \text { by symmetry }
\end{aligned}
$$


We have the conjugation formulas (see also (87))

$$
\begin{aligned}
& \mathcal{M}^{*} a_{x} \mathcal{M}=a_{x}(c)+a_{x}^{*}(u)+\sqrt{N} \phi(x):=b_{x}+\sqrt{N} \phi(x) \\
& \mathcal{M}^{*} a_{x}^{*} \mathcal{M}=a_{x}^{*}(\bar{c})+a_{x}(\bar{u})+\sqrt{N} \bar{\phi}(x):=b_{x}^{*}+\sqrt{N} \bar{\phi}(x)
\end{aligned}
$$

which implies the transformation of the monomial,

$$
\mathcal{M}^{*} \mathcal{P}\left(a^{*}, a\right) \mathcal{M}=\mathcal{P}\left(b^{*}+\sqrt{N} \bar{\phi}, b+\sqrt{N} \phi\right) .
$$

Now if $\mathcal{P}=a$ then, using (18),

$$
\left\langle\Omega, \mathcal{H}_{\text {red }}\left(b_{x}+\sqrt{N} \phi(x)\right) \Omega\right\rangle-\left\langle\Omega,\left(b_{x}+\sqrt{N} \phi(x)\right) \mathcal{H}_{\text {red }} \Omega\right\rangle=0 .
$$

The argument is similar if $\mathcal{P}=a a$ or $\mathcal{P}=a^{*} a$ since only the entries in the zeroth slot survive.

Based on this, we easily prove the following proposition.

Proposition 4.3. Under the assumptions of Lemma (4.2), the following equations hold

$$
\begin{aligned}
& \left(\frac{1}{i} \frac{\partial}{\partial t}-\Delta_{x_{1}}\right) \mathcal{L}_{0,1}\left(t, x_{1}\right) \\
& =-\int v_{N}\left(x_{1}-x_{2}\right) \mathcal{L}_{1,2}\left(t, x_{2} ; x_{1}, x_{2}\right) d x_{2} \\
& \left(\frac{1}{i} \frac{\partial}{\partial t}+\Delta_{x_{1}}-\Delta_{y_{1}}\right) \mathcal{L}_{1,1}\left(t, x_{1} ; y_{1}\right) \\
& =\int v_{N}\left(x_{1}-x_{2}\right) \mathcal{L}_{2,2}\left(t, x_{1}, x_{2} ; y_{1}, x_{2}\right) d x_{2}-\int v_{N}\left(y_{1}-y_{2}\right) \mathcal{L}_{2,2}\left(t, x_{1}, y_{2} ; y_{1}, y_{2}\right) d y_{2} \\
& \left(\frac{1}{i} \frac{\partial}{\partial t}-\Delta_{x_{1}}-\Delta_{x_{2}}+\frac{1}{N} v_{N}\left(x_{1}-x_{2}\right)\right) \mathcal{L}_{0,2}\left(t, x_{1}, x_{2}\right) \\
& =-\int v_{N}\left(x_{1}-y\right) \mathcal{L}_{1,3}\left(t, y ; x_{1}, x_{2}, y\right) d y-\int v_{N}\left(x_{2}-y\right) \mathcal{L}_{1,3}\left(t, y ; x_{1}, x_{2}, y\right) d y
\end{aligned}
$$

The equation (40b) is one of the BBGKY equations, but in our case $\mathcal{L}_{2,2}$ can be expressed in terms of the earlier matrices, see Lemma (4.4).

Proof. With $\mathcal{P}$ any monomial of degree one or two we know from Lemma (4.2) that

$$
\left\langle\Omega,\left[\mathcal{H}_{\text {red }}, \mathcal{M}^{*} \mathcal{P} \mathcal{M}\right] \Omega\right\rangle=0
$$


and for any of the corresponding matrices $\mathcal{L}$ we arrive at the equation,

$$
\frac{1}{i} \partial_{t} \mathcal{L}=\frac{1}{N^{\alpha}}\left\langle\Omega, \mathcal{M}^{*}[\mathcal{P}, \mathcal{H}] \mathcal{M} \Omega\right\rangle \quad, \quad \alpha=1 / 2,1 .
$$

We need to compute $[\mathcal{P}, \mathcal{H}]$ and for this purpose recall our original Hamiltonian,

$$
\mathcal{H}=\int d x d y\left\{\Delta_{x} \delta(x-y) a_{x}^{*} a_{y}\right\}-\frac{1}{2 N} \int d x d y\left\{v_{N}(x-y) a_{x}^{*} a_{y}^{*} a_{x} a_{x}\right\}
$$

Below we list the commutators with each mononomial:

$$
\begin{aligned}
{\left[a_{x_{1}}, \mathcal{H}\right] } & =\Delta_{x_{1}} a_{x_{1}}-\frac{1}{N} \int d y\left\{v_{N}\left(x_{1}-y\right) a_{y}^{*} a_{y}\right\} a_{x_{1}} \\
{\left[a_{x_{1}} a_{x_{2}}, \mathcal{H}\right] } & =\Delta_{x_{1}} a_{x_{1}} a_{x_{2}}+\Delta_{x_{2}} a_{x_{1}} a_{x_{2}}-\frac{1}{N} v_{N}\left(x_{1}-x_{2}\right) a_{x_{1}} a_{x_{2}} \\
& -\frac{1}{N} \int d z\left\{\left(v_{N}\left(x_{1}-z\right)+v\left(x_{2}-z\right)\right) a_{z}^{*} a_{z} a_{x_{1}} a_{x_{2}}\right\} \\
{\left[a_{x_{1}}^{*} a_{x_{2}}, \mathcal{H}\right] } & =a_{x_{1}}^{*} \Delta_{x_{2}} a_{x_{2}}-\left(\Delta_{x_{1}} a_{x_{1}}\right)^{*} a_{x_{2}} \\
& +\frac{1}{N} \int d z\left\{\left(v_{N}\left(x_{1}-z\right)-v_{N}\left(x_{2}-z\right)\right) a_{x_{1}}^{*} a_{z}^{*} a_{z} a_{x_{2}}\right\}
\end{aligned}
$$

from which we can derive the corresponding evolution equations for the $\mathcal{L}$ matrices:

$$
\begin{gathered}
\left\{\frac{1}{i} \partial_{t}-\Delta_{x_{1}}\right\} \mathcal{L}_{0,1}\left(t, x_{1}\right)=-\frac{1}{N^{3 / 2}} \int d y v_{N}\left(x_{1}-y\right)\left\{\left\langle\Omega, \mathcal{M}^{*} a_{y}^{*} a_{y} a_{x_{1}} \mathcal{M} \Omega\right\rangle\right\} \\
\quad\left\{\frac{1}{i} \partial_{t}-\Delta_{x_{1}}-\Delta_{x_{2}}+\frac{1}{N} v_{N}\left(x_{1}-x_{2}\right)\right\} \mathcal{L}_{0,2}\left(t, x_{1}, x_{2}\right)= \\
\quad-\frac{1}{N^{2}} \int d z\left\{\left(v_{N}\left(x_{1}-z\right)+v_{N}\left(x_{2}-z\right)\right)\left\langle\Omega, \mathcal{M}^{*} a_{z}^{*} a_{z} a_{x_{1}} a_{x_{2}} \mathcal{M} \Omega\right\rangle\right\}
\end{gathered}
$$

and finally

$$
\begin{aligned}
& \left\{\frac{1}{i} \partial_{t}+\Delta_{x_{1}}-\Delta_{x_{2}}\right\} \mathcal{L}_{1,1}\left(t, x_{1}, x_{2}\right)= \\
& +\frac{1}{N^{2}} \int d z\left\{\left(v_{N}\left(x_{1}-z\right)-v_{N}\left(x_{2}-z\right)\right)\left\langle\Omega, \mathcal{M}^{*} a_{x_{1}}^{*} a_{z}^{*} a_{z} a_{x_{2}} \mathcal{M} \Omega\right\rangle\right\}
\end{aligned}
$$

which implies the statement of the proposition. 
The proof of Theorem (4.1) is finished by computing the necessary matrices $\mathcal{L}$. We need the following (writing throughout this proof $[x]$ indicates freezing the variable $x$ ):

$$
\begin{aligned}
& \mathcal{M}^{*} a_{x_{1}} \mathcal{M} \Omega \\
& =\left(b_{x_{1}}+\sqrt{N} \phi\left(x_{1}\right)\right) \Omega=\left(\sqrt{N} \phi\left(\left[x_{1}\right]\right), u\left(y,\left[x_{1}\right]\right), 0,0 \ldots\right) \\
& \mathcal{M}^{*} a_{x_{1}} a_{x_{2}} \mathcal{M} \Omega= \\
& \left(b_{x_{1}}+\sqrt{N} \phi\left(x_{1}\right)\right)\left(b_{x_{2}}+\sqrt{N} \phi\left(x_{2}\right)\right) \Omega=\left(f_{0}, f_{1}, f_{2}, 0,0 \ldots\right)
\end{aligned}
$$

where the entries are :

$\left.\left.f_{0}\left(\left[x_{1}\right],\left[x_{2}\right]\right)=N \phi\left(\left[x_{1}\right]\right) \phi\left(\left[x_{2}\right]\right)+(u \circ c)\right)\left[x_{1}\right],\left[x_{2}\right]\right)=N \Lambda\left(\left[x_{1}\right],\left[x_{2}\right]\right)$

$f_{1}\left(y,\left[x_{1}\right],\left[x_{2}\right]\right)=\sqrt{N}\left[\phi\left(\left[x_{1}\right]\right) u\left(y,\left[x_{2}\right]\right)+\phi\left(\left[x_{2}\right]\right) u\left(y,\left[x_{1}\right]\right)\right]$

$f_{2}\left(y_{1}, y_{2},\left[x_{1}\right],\left[x_{2}\right]\right)=\frac{1}{\sqrt{2}}\left[u\left(y_{1},\left[x_{1}\right]\right) u\left(y_{2},\left[x_{2}\right]\right)+u\left(y_{2},\left[x_{1}\right]\right) u\left(y_{1},\left[x_{2}\right]\right)\right]$

and similarly,

$$
\begin{aligned}
& \mathcal{M}^{*} a_{x_{1}}^{*} a_{x_{2}} \mathcal{M} \Omega= \\
& \left(b_{x_{1}}^{*}+\sqrt{N} \bar{\phi}\left(x_{1}\right)\left(\left(b_{x_{2}}+\sqrt{N} \phi\left(x_{2}\right)\right) \Omega=\left(g_{0}, g_{1}, g_{2}, 0,0 \ldots\right)\right.\right. \\
& \quad \text { where the entries are : } \\
& \left.g_{0}\left[x_{1}\right],\left[x_{2}\right]\right)=N \bar{\phi}\left(\left[x_{1}\right]\right) \phi\left(\left[x_{2}\right]\right)+(\bar{u} \circ u)\left(\left[x_{1}\right],\left[x_{2}\right]\right)=N \Gamma\left(\left[x_{1}\right],\left[x_{2}\right]\right) \\
& g_{1}\left(y,\left[x_{1}\right],\left[x_{2}\right]\right)=\sqrt{N}\left[\bar{\phi}\left(\left[x_{1}\right]\right) u\left(y,\left[x_{2}\right]\right)+\bar{c}\left(y,\left[x_{1}\right]\right) \phi\left(\left[x_{2}\right]\right)\right] \\
& g_{2}\left(y_{1}, y_{2},\left[x_{1}\right],\left[x_{2}\right]\right)=\frac{1}{\sqrt{2}}\left[\bar{c}\left(y_{1},\left[x_{1}\right]\right) u\left(y_{2},\left[x_{2}\right]\right)+\bar{c}\left(y_{2},\left[x_{1}\right]\right) u\left(y_{1},\left[x_{2}\right]\right)\right]
\end{aligned}
$$

Based on this we easily compute 
Lemma 4.4. The $\mathcal{L}$ matrices are given by

$$
\begin{aligned}
& \mathcal{L}_{0,1}\left(t, x_{1}\right)=\frac{1}{\sqrt{N}}\left\langle\Omega,\left(b_{x_{1}}+\sqrt{N} \phi\left(x_{1}\right)\right) \Omega\right\rangle=\phi\left(x_{1}\right) \\
& \mathcal{L}_{0,2}\left(t, x_{1}, x_{2}\right)=\frac{1}{N}\left\langle\Omega,\left(b_{x_{1}}+\sqrt{N} \phi\left(x_{1}\right)\right)\left(b_{x_{2}}+\sqrt{N} \phi\left(x_{2}\right)\right) \Omega\right\rangle \\
& =\frac{1}{N}(u \circ c)\left(x_{1}, x_{2}\right)+\phi\left(x_{1}\right) \phi\left(x_{2}\right) \\
& =\frac{1}{2 N} \psi\left(t, x_{1}, x_{2}\right)+\phi\left(t, x_{1}\right) \phi\left(t, x_{2}\right)=\Lambda \\
& \text { where } \quad \psi=\operatorname{sh}(2 k)=2 u \circ c \\
& \mathcal{L}_{1,1}\left(t, x_{1} ; x_{2}\right)=\frac{1}{N}(\bar{u} \circ u)\left(x_{1}, x_{2}\right)+\bar{\phi}\left(x_{1}\right) \phi\left(x_{2}\right) \\
& =\frac{1}{2 N} \omega\left(t, x_{1}, x_{2}\right)+\bar{\phi}\left(t, x_{1}\right) \phi\left(t, x_{2}\right)=\Gamma \\
& \text { where } \quad \omega:=\operatorname{ch}(2 k)-1=2 \bar{u} \circ u \text {, } \\
& \mathcal{L}_{1,2}\left(x_{1} ; x_{2}, x_{3}\right)=\Gamma\left(x_{1}, x_{2}\right) \phi\left(x_{3}\right)+\frac{1}{2 N} \psi\left(x_{3}, x_{2}\right) \bar{\phi}\left(x_{1}\right)+\frac{1}{N} \bar{u} \circ u\left(x_{1}, x_{3}\right) \phi\left(x_{2}\right) \\
& \mathcal{L}_{2,2}\left(y_{1}, y_{2} ; x_{1}, x_{2}\right)=\bar{\Lambda}\left(y_{1}, y_{2}\right) \Lambda\left(x_{1}, x_{2}\right)+\frac{1}{N^{2}} \int \bar{f}_{1}\left(y, y_{1}, y_{2}\right) f_{1}\left(y, x_{1}, x_{2}\right) d y \\
& +\frac{1}{N^{2}} \int \bar{f}_{2}\left(y, z, y_{1}, y_{2}\right) f_{2}\left(y, z, x_{1}, x_{2}\right) d y d z \\
& \mathcal{L}_{1,3}\left(y_{1} ; x_{1}, x_{2}, x_{3}\right)=\bar{\Gamma}\left(x_{1}, y_{1}\right) \Lambda\left(x_{2}, x_{3}\right)+\frac{1}{N^{2}} \int \bar{g}_{1}\left(y, x_{1}, y_{1}\right) f_{1}\left(y, x_{2}, x_{3}\right) d y \\
& +\frac{1}{N^{2}} \int \bar{g}_{2}\left(y, z, x_{1}, y_{1}\right) f_{2}\left(y, z, x_{2}, x_{3}\right) d y d z
\end{aligned}
$$

where the integrals in the last two formulas can be trivially expressed in terms of $\psi$ and $\omega$, which in turn can be expressed in terms of $\phi, \Lambda$ and $\Gamma$.

Proof. All calculations are straightforward. For instance,

$$
\begin{aligned}
& \mathcal{L}_{1,2}\left(x_{1} ; x_{2}, x_{3}\right)=\frac{1}{N^{3 / 2}}\left\langle\Omega, \mathcal{M}^{*} a_{x_{1}}^{*} a_{x_{2}} a_{x_{3}} \mathcal{M} \Omega\right\rangle=\frac{1}{N^{3 / 2}}\left\langle\Omega, \mathcal{M}^{*} a_{x_{1}}^{*} a_{x_{2}} \mathcal{M M}^{*} a_{x_{3}} \mathcal{M} \Omega\right\rangle \\
& =\frac{1}{N^{3 / 2}}\left\langle\mathcal{M}^{*} a_{x_{2}}^{*} a_{x_{1}} \mathcal{M} \Omega, \mathcal{M}^{*} a_{x_{3}} \mathcal{M} \Omega\right\rangle \\
& =\frac{1}{N} \bar{g}_{0}\left(x_{2}, x_{1}\right) \phi\left(x_{3}\right)+\int \bar{g}_{1}\left(y, x_{2}, x_{1}\right) u\left(y, x_{3}\right) d y \\
& =\Gamma\left(x_{1}, x_{2}\right) \phi\left(x_{3}\right)+\frac{1}{N} u \circ c\left(x_{3}, x_{2}\right) \bar{\phi}\left(x_{1}\right)+\frac{1}{N} \bar{u} \circ u\left(x_{1}, x_{3}\right) \phi\left(x_{2}\right) \\
& =\Gamma\left(x_{1}, x_{2}\right) \phi\left(x_{3}\right)+\frac{1}{2 N} \operatorname{sh}(2 k)\left(x_{3}, x_{2}\right) \bar{\phi}\left(x_{1}\right)+\frac{1}{N} \bar{u} \circ u\left(x_{1}, x_{3}\right) \phi\left(x_{2}\right)
\end{aligned}
$$




$$
\begin{aligned}
& \mathcal{L}_{2,2}\left(y_{1}, y_{2} ; x_{1}, x_{2}\right)=\frac{1}{N^{2}}\left\langle\mathcal{M}^{*} a_{y_{1}} a_{y_{2}} \mathcal{M} \Omega, \mathcal{M}^{*} a_{x_{1}} a_{x_{2}} \mathcal{M} \Omega\right\rangle \\
& =\frac{1}{N^{2}} \bar{f}_{0}\left(y_{1}, y_{2}\right) f_{0}\left(x_{1}, x_{2}\right)+\frac{1}{N^{2}} \int \bar{f}_{1}\left(y, y_{1}, y_{2}\right) f_{1}\left(y, x_{1}, x_{2}\right) d y \\
& +\frac{1}{N^{2}} \int \bar{f}_{2}\left(y, z, y_{1}, y_{2}\right) f_{2}\left(y, z, x_{1}, x_{2}\right) d y d z
\end{aligned}
$$

With these ingredients at hand we proceed to write down the evolution of $\mathcal{L}_{1}=\phi$ :

$$
\begin{aligned}
& \left\{\frac{1}{i} \partial_{t}-\Delta_{x_{1}}+\int d y\left\{v_{N}\left(x_{1}-y\right) \Gamma(y, y)\right\}\right\} \phi\left(x_{1}\right)= \\
& -\frac{1}{2 N} \int d y\left\{v_{N}\left(x_{1}-y\right) \phi(y) \omega\left(y, x_{1}\right)+v_{N}\left(x_{1}-y\right) \bar{\phi}(y) \psi\left(x_{1}, y\right)\right\}
\end{aligned}
$$

and we can eliminate $\psi$ and $\omega$ by the substitution,

$$
\begin{aligned}
& \omega=2 N(\Gamma-\bar{\phi} \otimes \phi) \\
& \psi=2 N(\Lambda-\phi \otimes \phi)
\end{aligned}
$$

so that we have a system involving only $\Lambda, \Gamma$ and $\phi$ matrices.

The evolution of $\Lambda$ is given by the expression below:

$$
\begin{aligned}
& \left\{\frac{1}{i} \partial_{t}-\Delta_{x_{1}}-\Delta_{x_{2}}+\frac{1}{N} v\left(x_{1}-x_{2}\right)+\int d y\left\{\left(v_{N}\left(x_{1}-y\right)+v_{N}\left(x_{2}-y\right)\right) \Gamma(y, y)\right\}\right\} \Lambda= \\
& -\frac{1}{2 N} \int d y\left\{\left(v_{N}\left(x_{1}-y\right)+v_{N}\left(x_{2}-y\right)\right) \phi(y)\left(\omega\left(y, x_{1}\right) \phi\left(x_{2}\right)+\omega\left(y, x_{2}\right) \phi\left(x_{1}\right)\right)\right\} \\
& -\frac{1}{2 N} \int d y\left\{\left(v_{N}\left(x_{1}-y\right)+v_{N}\left(x_{2}-y\right)\right) \bar{\phi}(y)\left(\psi\left(x_{1}, y\right) \phi\left(x_{2}\right)+\psi\left(x_{2}, y\right) \phi\left(x_{1}\right)\right)\right\} \\
& -\frac{1}{4 N^{2}} \int d y\left\{\left(v_{N}\left(x_{1}-y\right)+v_{N}\left(x_{2}-y\right)\right)\left(\psi\left(x_{1}, y\right) \omega\left(y, x_{2}\right)+\omega\left(y, x_{1}\right) \psi\left(x_{2}, y\right)\right)\right\}
\end{aligned}
$$

Finally the evolution of $\Gamma$ is given by:

$$
\begin{aligned}
& \left\{\frac{1}{i} \partial_{t}+\Delta_{x_{1}}-\Delta_{x_{2}}\right\} \Gamma=\int d y\left\{\left(v_{N}\left(x_{1}-y\right)-v_{N}\left(x_{2}-y\right)\right) \bar{\Lambda}\left(x_{1}, y\right) \Lambda\left(y, x_{2}\right)\right\}+ \\
& \frac{1}{2 N} \int d y\left\{\left(v_{N}\left(x_{1}-y\right)-v_{N}\left(x_{2}-y\right)\right)\left(\phi(y) \omega\left(y, x_{2}\right) \bar{\phi}\left(x_{1}\right)+\bar{\phi}(y) \omega\left(x_{1}, y\right) \phi\left(x_{2}\right)\right)\right\}+ \\
& \frac{1}{2 N} \int d y\left\{\left(v_{N}\left(x_{1}-y\right)-v_{N}\left(x_{2}-y\right)\right)\left(|\phi(y)|^{2} \omega\left(x_{1}, x_{2}\right)+\omega(y, y) \bar{\phi}\left(x_{1}\right) \phi\left(x_{2}\right)\right)\right\}+ \\
& \frac{1}{4 N^{2}} \int d y\left\{\left(v\left(x_{1}-y\right)-v\left(x_{2}-y\right)\right)\left(\omega\left(x_{1}, y\right) \omega\left(y, x_{2}\right)+\omega(y, y) \omega\left(x_{1}, x_{2}\right)\right)\right\}
\end{aligned}
$$


If we substitute $\psi=2 N(\Lambda-\phi \otimes \phi)$ and $\omega=2 N(\Gamma-\bar{\phi} \otimes \phi)$ we obtain the equations of Theorem (4.1).

Remark 4.5. Using the ideas in this section one can easily show that the expected number of particles for our approximation, $\langle\mathcal{M} \Omega, \mathcal{N} \mathcal{M} \Omega\rangle$ (where $\mathcal{N}=\int a_{x}^{*} a_{x} d x$ ), as well as the energy $\langle\mathcal{M} \Omega, \mathcal{H} \mathcal{M} \Omega\rangle$ are constant in time. This provides an easier proof of some of the results of section 8 of [24].

\section{Estimates}

In this paper, $\mathbf{S}$ and $\mathbf{S}_{ \pm}$stand for the pure differential operators, so that the operators of Theorem (2.4) are $\tilde{\mathbf{S}}=\mathbf{S}+$ potential terms, $\tilde{\mathbf{W}}=\mathbf{S}_{ \pm}+$potential terms

$\mathbf{S}=\frac{1}{i} \partial_{t}-\Delta$ (in $6+1$ or $3+1$ dimensions, as will be clear from the context)

$\mathbf{S}_{ \pm}=\frac{1}{i} \partial_{t}-\Delta_{x}+\Delta_{y}$

and potential terms are given by composition with $v_{N}(x-y) \Gamma(x, y)$ and multiplication by $v_{N} * \operatorname{Tr} \Gamma$.

The symbol of $\mathbf{S}$ is $\tau+|\xi|^{2}$ or $\tau+|\xi|^{2}+|\eta|^{2}$, depending on dimensions, and the symbol of $S_{ \pm}$is $\tau+|\xi|^{2}-|\eta|^{2}$. We will use the following norms:

$$
\begin{aligned}
& \|f\|_{X^{\delta}}=\left\|\left(1+\left.|\tau+| \xi\right|^{2} \mid\right)^{\delta} \hat{f}(\tau, \xi)\right\|_{L^{2}} \\
& \|f\|_{X_{S}^{\delta}}=\left\|\left(1+\left.|\tau+| \xi\right|^{2}+|\eta|^{2} \mid\right)^{\delta} \hat{f}(\tau, \xi, \eta)\right\|_{L^{2}} \\
& \|f\|_{X_{W}^{\delta}}=\left\|\left(1+\left.|\tau+| \xi\right|^{2}-|\eta|^{2} \mid\right)^{\delta} \hat{f}(\tau, \xi, \eta)\right\|_{L^{2}}
\end{aligned}
$$

and refer to [43, section 2.6 for their history and properties. Of special importance is the following result: (proposition 2.12 in [43]): if $\mathbf{S} u=f$, $\delta>0$ and $\chi(t)$ is a fixed $C_{0}^{\infty}$, cut-off function, then

$$
\|\chi(t) u\|_{X^{\frac{1}{2}+\delta}} \lesssim \chi, \delta\|u(0, \cdot)\|_{L^{2}}+\|f\|_{X^{-\frac{1}{2}+\delta}}
$$

For the rest of the paper, we will use the standard notation $A \lesssim_{\chi, \delta} B$ to mean "there exists a constant $C$ depending of $\chi$ and $\delta$ such that $A \leq C B$.

We will also use freely the general principle that if an $L^{p}$ estimate holds for solutions to the homogeneous equation, it also holds in $X^{1 / 2+\delta}$ spaces, see Lemma 2.9 in 43 .

To get started, fix $w \in \mathcal{S}$ such that $|\hat{v}| \leq \hat{w}$ and fix $\epsilon>0$ depending on $\beta<2 / 3$ so that $<\nabla_{x}>^{\frac{1}{2}+\epsilon}<\nabla_{y}>^{\frac{1}{2}+\epsilon} \frac{1}{N} w_{N}(x-y)$, which is a 
function of $x-y$, satisfies

$$
\left\|<\nabla_{x}>^{\frac{1}{2}+\epsilon}<\nabla_{y}>^{\frac{1}{2}+\epsilon} \frac{1}{N} w_{N}\right\|_{L^{6 / 5}(d(x-y)} \leq \frac{C}{N^{\text {small power }}}
$$

The basic space-time collapsing estimates, in the spirit of [31], are:

Lemma 5.1. If $\mathbf{S} \Lambda=0$ then

$$
\begin{aligned}
& \left\|\left.\left|\Lambda(t, x, x)\left\|_{L^{2}(d t d x)} \lesssim\right\|\right| \nabla\right|_{x-y} ^{1 / 2} \Lambda_{0}(x, y)\right\|_{L^{2}(d x d y)} \\
& \left\||\nabla|_{x}^{1 / 2} \Lambda(t, x, x)\right\|_{L^{2}(d t d x)} \lesssim\left\||\nabla|_{x}^{1 / 2}|\nabla|_{y}^{1 / 2} \Lambda_{0}(x, y)\right\|_{L^{2}(d x d y)}
\end{aligned}
$$

As a consequence, if $\mathbf{S} \Lambda=F$ and $\delta>0$, then

$$
\begin{aligned}
& \sup _{z}\left\|<\nabla_{x}>^{\frac{1}{2}+\epsilon} \chi(t) \Lambda(t, x, x+z)\right\|_{L^{2}(d t d x)} \\
& \lesssim_{\delta}\left\|<\nabla_{x}>^{\frac{1}{2}+\epsilon}<\nabla_{y}>^{\frac{1}{2}+\epsilon} \chi(t) \Lambda\right\|_{X_{S}^{1 / 2+\delta}} \\
& \lesssim_{\delta}\left\|<\nabla_{x}>^{\frac{1}{2}+\epsilon}<\nabla_{y}>^{\frac{1}{2}+\epsilon} \Lambda_{0}(x, y)\right\|_{L^{2}(d x d y)} \\
& +\left\|<\nabla_{x}>^{\frac{1}{2}+\epsilon}<\nabla_{y}>^{\frac{1}{2}+\epsilon} F\right\|_{X_{S}^{-1 / 2+\delta}}
\end{aligned}
$$

If $\mathbf{S}_{ \pm} \Gamma=0$, then

$$
\begin{aligned}
& \left\|\left|\nabla_{x}\right|^{\frac{1}{2}+\epsilon} \Gamma(t, x, x)\right\|_{L^{2}(d t d x)}+\left\|\left|\nabla_{x}\right|^{1 / 2} \Gamma(t, x, x)\right\|_{L^{2}(d t d x)} \\
& \lesssim_{\epsilon}\left\|<\nabla_{x}>^{\frac{1}{2}+\epsilon}<\nabla_{y}>^{\frac{1}{2}+\epsilon} \Gamma_{0}(x, y)\right\|_{L^{2}(d x d y)}
\end{aligned}
$$

As a consequence, if $\mathbf{S}_{ \pm} \Gamma=F$, then

$$
\begin{aligned}
& \sup _{z}\left\|\left|\nabla_{x}\right|^{\frac{1}{2}+\epsilon} \chi(t) \Gamma(t, x, x+z)\right\|_{L^{2}(d t d x)}+\sup _{z}\left\|\left|\nabla_{x}\right|^{1 / 2} \chi(t) \Gamma(t, x, x+z)\right\|_{L^{2}(d t d x)} \\
& \lesssim_{\epsilon, \delta}<\nabla_{x}>^{\frac{1}{2}+\epsilon}<\nabla_{y}>^{\frac{1}{2}+\epsilon} \chi(t) \Gamma \|_{X_{W}^{1 / 2+\delta}} \\
& \lesssim_{\epsilon, \delta}\left\|<\nabla>_{x}^{\frac{1}{2}+\epsilon}<\nabla_{y}>^{\frac{1}{2}+\epsilon} \Gamma_{0}(x, y)\right\|_{L^{2}(d x d y)} \\
& +\left\|<\nabla_{x}>^{\frac{1}{2}+\epsilon}<\nabla_{y}>^{\frac{1}{2}+\epsilon} F\right\|_{X_{W}^{-1 / 2+\delta}}
\end{aligned}
$$

Remark 5.2. Notice that the estimates for $\Lambda$ are different from those for $\Gamma$ at low frequencies. We cannot estimate $\|\Gamma(t, x, x)\|_{L^{2}(d t d x)}$. The "lowest" derivative we can estimate in (55) is $\left\|\left|\nabla_{x}\right|^{1 / 2} \Gamma(t, x, x)\right\|_{L^{2}(d t d x)}$. 
Proof. The same method, inspired by [30, works for both. Let $\widetilde{\Lambda}$ denote the space-time Fourier transform of $\Lambda$. For (52)

$$
\begin{aligned}
& |\Lambda(t, x, x)(\tau, \xi)|^{2} \\
& \lesssim \int \delta\left(\tau-|\xi-\eta|^{2}-|\xi+\eta|^{2}\right) \frac{1}{|\eta|} d \eta \\
& \int \delta\left(\tau-|\xi-\eta|^{2}-|\xi+\eta|^{2}\right)\left|\widetilde{\nabla_{x-y}^{\frac{1}{2}} \Lambda_{0}}(\xi-\eta, \xi+\eta)\right|^{2} d \eta
\end{aligned}
$$

In order to prove the estimate, we must show

$$
\sup _{\tau, \xi} \int \delta\left(\tau-|\xi|^{2}-|\eta|^{2}\right) \frac{1}{|\eta|} d \eta \lesssim 1
$$

which is obvious. For (53)

$$
\begin{aligned}
& \left|\nabla_{x}^{1 / 2} \widehat{\Lambda(t, x, x)}(\tau, \xi)\right|^{2} \\
& \lesssim \int \delta\left(\tau-|\xi-\eta|^{2}-|\xi+\eta|^{2}\right) \frac{|\xi|}{|\xi-\eta||\xi+\eta|} d \eta \\
& \quad \int \delta\left(\tau-|\xi-\eta|^{2}-|\xi+\eta|^{2}\right)\left|\nabla_{x}^{\frac{1}{2} \nabla_{y}^{\frac{1}{2}}} \Lambda_{0}(\xi-\eta, \xi+\eta)\right|^{2} d \eta
\end{aligned}
$$

In order to prove the estimate, we must show

$$
\sup _{\tau, \xi} \int \delta\left(\tau-|\xi|^{2}-|\eta|^{2}\right) \frac{|\xi|}{|\xi-\eta||\xi+\eta|} d \eta \lesssim 1
$$

Without loss of generality, consider the region $|\xi-\eta| \leq|\xi+\eta|$. If $|\xi-\eta| \sim|\xi+\eta|, \frac{|\xi|}{|\xi-\eta||\xi+\eta|} \lesssim \frac{1}{|\eta|}$ and the integral can be evaluated in polar coordinates. If $|\xi-\eta|<<|\xi+\eta|$ then $|\xi| \sim|\eta|$ Writing $\frac{|\xi|}{|\xi-\eta||\xi+\eta|} \lesssim \frac{1}{|\xi-\eta|} \lesssim \frac{1}{|\eta| \sqrt{1-\cos (\theta)}}$ where $\theta$ is the angle between $\xi$ and $\eta$, we estimate

$$
\sup _{\tau} \int_{0}^{\pi} \int \delta\left(\tau-\rho^{2}\right) \frac{1}{\rho \sqrt{1-\cos (\theta)}} \rho^{2} d \rho \sin (\theta) d \theta \lesssim 1
$$

For (55)

$$
\begin{aligned}
& \left.|| \nabla_{x}\right|^{\frac{1+\epsilon}{2} \Gamma(t, x, x)}(\tau, \xi)|=c| \int \delta\left(\tau-|\xi-\eta|^{2}+|\xi+\eta|^{2}\right)|\xi|^{\frac{1+\epsilon}{2}} \hat{\Gamma}_{0}(\xi-\eta, \xi+\eta) d \eta \mid \\
& \lesssim \int \delta\left(\tau-|\xi-\eta|^{2}+|\xi+\eta|^{2}\right)\left(|\xi-\eta|^{\frac{\epsilon}{2}}+|\xi+\eta|^{\frac{\epsilon}{2}}\right)|\xi|^{\frac{1}{2}}\left|\hat{\Gamma}_{0}(\xi-\eta, \xi+\eta)\right| d \eta
\end{aligned}
$$


Here we use the straightforward estimate

$$
I=\sup _{\tau, \xi} \int \delta(\tau-\xi \cdot \eta) \frac{|\xi||\xi \pm \eta|^{\epsilon}}{<\xi-\eta>^{1+\epsilon}<\xi+\eta>^{1+\epsilon}} d \eta \lesssim 1
$$

To prove this, take $\xi=(|\xi|, 0,0)$. Then

$$
\begin{aligned}
& \int \delta(\tau-\xi \cdot \eta) \frac{|\xi||\xi-\eta|^{\epsilon}}{<\xi-\eta>^{1+\epsilon}<\xi+\eta>^{1+\epsilon}} d \eta \\
& =\int \delta\left(\tau-|\xi| \eta_{1}\right) \frac{|\xi|}{<\xi-\eta><\xi+\eta>^{1+\epsilon}} d \eta \lesssim \int_{\mathbb{R}^{2}} \frac{1}{<\eta_{2,3}>^{2+\epsilon}} d \eta_{2} d \eta_{3} \lesssim 1
\end{aligned}
$$

Next, we record some Strichartz type estimates

Lemma 5.3. The following estimate holds

$$
\begin{array}{r}
\left\|e^{i t\left(\Delta_{x} \pm \Delta_{y}\right)} f\right\|_{L^{2}(d t) L^{6}(d x) L^{2}(d y)} \lesssim\|f\|_{L^{2} .} \\
\left\|e^{i t\left(\Delta_{x} \pm \Delta_{y}\right)} f\right\|_{L^{2}(d t) L^{\infty}(d x) L^{2}(d y)} \lesssim\left\|<\nabla_{x}>^{1 / 2+\epsilon} f\right\|_{L^{2} .}
\end{array}
$$

and, as a consequence

$$
\|F\|_{L^{2}(d t) L^{6}(d x) L^{2}(d y)} \lesssim_{\delta}\|F\|_{X^{1 / 2+\delta}}
$$

where $X$ can be either $X_{S}$ or $X_{W}$.

Proof. We argue as follows:

$$
\begin{aligned}
& \|\| e^{i t\left(\Delta_{x} \pm \Delta_{y}\right)} f\left\|_{L^{2}(d y)}\right\|_{L^{2}(d t) L^{6}(d x)}=\|\| e^{i t \Delta_{x}} f\left\|_{L^{2}(d y)}\right\|_{L^{2}(d t) L^{6}(d x)} \\
& \lesssim\|\| e^{i t \Delta_{x}} f\left\|_{L^{2}(d t) L^{6}(d x)}\right\|_{L^{2}(d y)} \\
& \lesssim\|\| e^{i t \Delta_{x}} f\left\|_{L^{2}(d t) L^{6}(d x)}\right\|_{L^{2}(d y)} \lesssim\|f\|_{L^{2}(d x d y)} \quad 3+1 \text { end-point Strichartz [27] }
\end{aligned}
$$

The proof of (57) is similar, using Sobolev. This type of estimate has first appeared, we believe, in [8]. Versions of it were used in [10], 11], 33 .

We will need the following refinements. Notice that for $\mathbf{S}$ we can choose $x, y$ coordinates or $x+y, x-y$ coordinates, but this is not possible for $\mathbf{S}_{ \pm}$.

Lemma 5.4. For each $0<\delta<\frac{1}{2}$ there exists $6 / 5+>6 / 5$ a number which can be chosen arbitrarily close to $6 / 5$ if $\delta$ is small such that the following estimate holds

$$
\|\Lambda\|_{X_{S}^{-\frac{1}{2}+\delta}} \lesssim \delta\|\Lambda\|_{L^{2} L^{6 / 5+}(x-y) L^{2}(x+y)}
$$


Proof. This is proved by interpolating the estimate dual to (59)

$$
\|\Lambda\|_{X_{S}^{-\frac{1}{2}-\delta}} \lesssim\|\Lambda\|_{L^{2} L^{6 / 5}(x-y) L^{2}(x+y)}
$$

with

$$
\|\Lambda\|_{X_{S}^{-\frac{1}{2}+\frac{1}{2}}}=\|\Lambda\|_{L^{2} L^{2}(x-y) L^{2}(x+y)}
$$

Also we will need the closely related

Lemma 5.5. For each $0<\delta<\frac{1}{2}$ there exist numbers $2-, 6 / 5+$ arbitrarily close to $2,6 / 5$ if $\delta$ is close to 0 , so that

$$
\|F\|_{X_{W}^{-1 / 2+\delta}} \lesssim_{\delta}\|F\|_{L^{2-}(d t) L^{6 / 5+}(d x) L^{2} d(y)}
$$

Proof. We start with

$\|F\|_{L^{2+}(d t) L^{6-}(d x) L^{2}(d y)} \lesssim\|F\|_{X_{W}^{1 / 2+\delta}}$ (interpolate (159) with energy estimates) $\|F\|_{X_{W}^{-1 / 2-\delta}} \lesssim\|F\|_{L^{2-}(d t) L^{6 / 5+}(d x) L^{2}(d y)}$ (dual estimates)

and interpolate with the trivial estimate

$$
\|F\|_{X_{W}^{-1 / 2+1 / 2}}=\|F\|_{L^{2}(d t) L^{2}(d x d y)}
$$

we get (64).

Finally, we have one more estimate along the same lines

Lemma 5.6. If $\mathbf{S} \Lambda=0$ then

$$
\|\Lambda\|_{L^{2}(d t) L^{\infty}(d(x-y)) L^{2}(d(x+y))} \lesssim\left\|<\nabla_{x}>^{\frac{1}{2}+\epsilon}<\nabla_{y}>^{\frac{1}{2}+\epsilon} \Lambda_{0}\right\|_{L^{2}(d x d y)}
$$

and, as a consequence, if $\mathbf{S} \Lambda=F$ then

$$
\begin{aligned}
& \|\chi(t) \Lambda\|_{L^{2}(d t) L^{\infty}(d(x-y)) L^{2}(d(x+y))} \\
& \lesssim\left\|<\nabla_{x}>^{\frac{1}{2}+\epsilon}<\nabla_{y}>^{\frac{1}{2}+\epsilon} \Lambda_{0}\right\|_{L^{2}(d x d y)}+\left\|<\nabla_{x}>^{\frac{1}{2}+\epsilon}<\nabla_{y}>^{\frac{1}{2}+\epsilon} F\right\|_{X_{S}^{-\frac{1}{2}+\delta}}
\end{aligned}
$$

Proof. Writing (58) in $x+y, x-y$ coordinates

$$
\begin{aligned}
& \left\|e^{i t\left(\Delta_{x}+\Delta_{y}\right)} \Lambda_{0}\right\|_{L^{2}(d t) L^{\infty}(d(x-y)) L^{2}(d(x+y))} \\
& \lesssim\left\|<\nabla_{x-y}>^{\frac{1}{2}+\epsilon} \Lambda_{0}\right\|_{L^{2}(d x d y)} \lesssim\left\|<\nabla_{x}>^{\frac{1}{2}+\epsilon}<\nabla_{y}>^{\frac{1}{2}+\epsilon} \Lambda_{0}\right\|_{L^{2}(d x d y)}
\end{aligned}
$$


Note that here we are forced to use $\langle\nabla\rangle$ rather than $\nabla$.

With the help of Lemma (5.6) we can estimate solutions to

$$
\begin{aligned}
& \left(\mathbf{S}+\frac{1}{N} v_{N}(x-y)\right) \Lambda=F \\
& \Lambda(0, \cdot)=\Lambda_{0}(\cdot)
\end{aligned}
$$

The next proposition is the key estimate of our paper.

Proposition 5.7. If $\Lambda$ satisfies (65), $\beta<2 / 3$, and $\chi(t)$ is a smooth cut-off function which is 1 on $[0,1], \delta$ is sufficiently small, and $N \geq N_{0}$ is sufficiently large, then

$$
\begin{aligned}
& \left\|<\nabla_{x}>^{\frac{1}{2}+\epsilon}<\nabla_{y}>^{\frac{1}{2}+\epsilon} \chi(t) \Lambda\right\|_{X_{S}^{\frac{1}{2}+\delta}} \\
& \lesssim\left\|<\nabla_{x}>^{\frac{1}{2}+\epsilon}<\nabla_{y}>^{\frac{1}{2}+\epsilon} \Lambda_{0}(x, y)\right\|_{L^{2}(d x d y)}+\left\|<\nabla_{x}>^{\frac{1}{2}+\epsilon}<\nabla_{y}>^{\frac{1}{2}+\epsilon} F\right\|_{X_{S}^{-\frac{1}{2}+\delta}}
\end{aligned}
$$

and thus, by Lemma (5.1),

$$
\begin{aligned}
& \sup _{z}\left\|<\nabla_{x}>^{\frac{1}{2}+\epsilon} \chi(t) \Lambda(t, x+z, x)\right\|_{L^{2}(d t d x)} \\
& \lesssim\left\|<\nabla_{x}>^{\frac{1}{2}+\epsilon}<\nabla_{y}>^{\frac{1}{2}+\epsilon} \Lambda_{0}(x, y)\right\|_{L^{2}(d x d y)}+\left\|<\nabla_{x}>^{\frac{1}{2}+\epsilon}<\nabla_{y}>^{\frac{1}{2}+\epsilon} F\right\|_{X_{S}^{-\frac{1}{2}+\delta}}
\end{aligned}
$$

Similar estimates hold for $\left(\frac{\partial}{\partial t}\right)^{i} \nabla_{x+y}^{j}(\chi(t) \Lambda)$ because these derivatives commute with the potential.

Proof. Recall we introduces a potential $w \in \mathcal{S}$ such that $\hat{w} \geq|\hat{v}|$. For (66) , we use another cut-off function $\chi_{1}(t)$ which is identically 1 on the support of $\chi$ and notice that the solution of (65) agrees with that of

$$
\begin{aligned}
& \mathbf{S} \Lambda+\frac{1}{N} v_{N} \chi_{1}(t) \Lambda=F \\
& \Lambda(0, \cdot)=\Lambda_{0}(\cdot)
\end{aligned}
$$

on the support of $\chi$. So we work with the solution of (68). Also, for technical reasons, we define $\left\lfloor\chi_{1}(t) \Lambda\right\rfloor$ to be the inverse Fourier transform of the absolute value of the (space-time) Fourier transform of $\chi_{1}(t) \Lambda$. Then

$$
\begin{aligned}
& \left\|<\nabla_{x}>^{\frac{1}{2}+\epsilon}<\nabla_{y}>^{\frac{1}{2}+\epsilon} \chi_{1}(t) \Lambda\right\|_{X_{S}^{\frac{1}{2}+\delta}} \\
& \lesssim\left\|<\nabla_{x}>^{\frac{1}{2}+\epsilon}<\nabla_{y}>^{\frac{1}{2}+\epsilon}\left(\frac{1}{N} v_{N} \chi_{1}(t) \Lambda\right)\right\|_{X_{S}^{-\frac{1}{2}+\delta}} \\
& +\left\|<\nabla_{x}>^{\frac{1}{2}+\epsilon}<\nabla_{y}>^{\frac{1}{2}+\epsilon} \Lambda_{0}(x, y)\right\|_{L^{2}(d x d y)}+\left\|<\nabla_{x}>^{\frac{1}{2}+\epsilon}<\nabla_{y}>^{\frac{1}{2}+\delta} F\right\|_{X_{S}^{-\frac{1}{2}+\delta}}
\end{aligned}
$$


We plan to absorb (70) in the LHS of (69) . The reason we introduced $w$ and $\left\lfloor\chi_{1}(t) \Lambda\right\rfloor$, which have non-negative Fourier transforms, is to have the following cheap substitute for the Leibnitz rule:

$$
\begin{aligned}
& \left\|<\nabla_{x}>^{\frac{1}{2}+\epsilon}<\nabla_{y}>^{\frac{1}{2}+\epsilon}\left(\frac{1}{N} \chi_{1}(t) v_{N} \Lambda\right)\right\|_{X_{S}^{-\frac{1}{2}+\delta}} \\
& \lesssim\left\|\left(<\nabla_{x}>^{\frac{1}{2}+\epsilon}<\nabla_{y}>^{\frac{1}{2}+\delta} \frac{1}{N} w_{N}\right)\left\lfloor\chi_{1}(t) \Lambda\right\rfloor\right\|_{X_{S}^{-\frac{1}{2}+\delta}} \\
& +\left\|\left(<\nabla_{x}>^{\frac{1}{2}+\epsilon} \frac{1}{N} w_{N}\right)<\nabla_{y}>^{\frac{1}{2}+\epsilon}\left\lfloor\chi_{1}(t) \Lambda\right\rfloor\right\|_{X_{S}^{-\frac{1}{2}+\delta}} \\
& +\left\|\left(<\nabla_{y}>^{\frac{1}{2}+\epsilon} \frac{1}{N} w_{N}\right)<\nabla_{x}>^{\frac{1}{2}+\epsilon}\left\lfloor\chi_{1}(t) \Lambda\right\rfloor\right\|_{X_{S}^{-\frac{1}{2}+\delta}} \\
& +\left\|\frac{1}{N} w_{N}<\nabla_{x}>^{\frac{1}{2}+\epsilon}<\nabla_{y}>^{\frac{1}{2}+\epsilon}\left\lfloor\chi_{1}(t) \Lambda\right\rfloor\right\|_{X_{S}^{-\frac{1}{2}+\delta}}
\end{aligned}
$$

For the most singular term, (71a),$\left\langle\nabla_{x}>^{\frac{1}{2}+\epsilon}<\nabla_{y}>^{\frac{1}{2}+\epsilon} \frac{1}{N} w_{N}(x-y)\right.$ is a function of $x-y$ and we recall $\epsilon$ was chosen so that

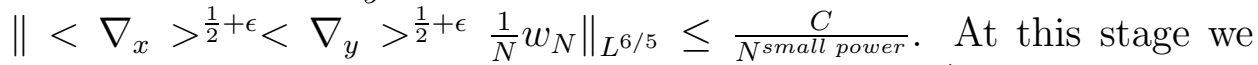
insist $\delta$ is so small that the corresponding number $6 / 5+$ also satisfies $\left\|<\nabla_{x}>^{\frac{1}{2}+\epsilon}<\nabla_{y}>^{\frac{1}{2}+\epsilon} \frac{1}{N} w_{N}\right\|_{L^{6 / 5+}} \leq \frac{C}{N^{\text {small power }}}$. We estimate, using (60) and Lemma (5.6)

$$
\begin{aligned}
& \left\|\left(<\nabla_{x}>^{\frac{1}{2}+\epsilon}<\nabla_{y}>^{\frac{1}{2}+\epsilon} \frac{1}{N} w_{N}\right)\left\lfloor\chi_{1}(t) \Lambda\right\rfloor\right\|_{X^{-\frac{1}{2}+\delta}} \\
& \lesssim\left\|\left(\nabla_{x}^{\frac{1}{2}+\epsilon} \nabla_{y}^{\frac{1}{2}+\epsilon} \frac{1}{N} w_{N}\right)\left\lfloor\chi_{1}(t) \Lambda\right\rfloor\right\|_{L^{2}(d t) L^{6 / 5+}(d(x-y)) L^{2}(d(x+y))} \\
& \lesssim\left\|\nabla_{x}^{\frac{1}{2}+\epsilon} \nabla_{y}^{\frac{1}{2}+\epsilon} \frac{1}{N} w_{N}\right\|_{L^{6 / 5+}(d(x-y))}\left\|\left\lfloor\chi_{1}(t) \Lambda\right\rfloor\right\|_{L^{2}(d t) L^{\infty}(d(x-y)) L^{2}(d(x+y))} \\
& \lesssim \frac{1}{N^{\text {small power }}}\left\|<\nabla_{x}>^{\frac{1}{2}+\epsilon}<\nabla_{y}>^{\frac{1}{2}+\epsilon}\left\lfloor\chi_{1}(t) \Lambda\right\rfloor\right\|_{X^{\frac{1}{2}+\delta}} \\
& =\frac{1}{N^{\text {small power }}}\left\|<\nabla_{x}>^{\frac{1}{2}+\epsilon}<\nabla_{y}>^{\frac{1}{2}+\epsilon} \chi_{1}(t) \Lambda\right\|_{X^{\frac{1}{2}+\delta}}
\end{aligned}
$$

This can be absorbed in the LHS of (69). The other terms are easier.

While we will use the $X$ spaces as tools in our proofs, the actual norms in which we prove well-posedness are $L^{p}$ norms defined for some 
$0<T \leq 1$.

$$
\begin{aligned}
\mathbf{N}_{T}(\Lambda)= & \sup _{z}\left\|<\nabla_{x}>^{\frac{1}{2}+\epsilon} \Lambda(t, x+z, x)\right\|_{L^{2}\left([0, T] \times \mathbb{R}^{3}\right)} \\
& +\left\|<\nabla_{x}>^{\frac{1}{2}+\epsilon}<\nabla_{y}>^{\frac{1}{2}+\epsilon} \Lambda(t, x, y)\right\|_{L^{\infty}\left([0, T] \times L^{2}\left(\mathbb{R}^{6}\right)\right)} \\
\dot{\mathbf{N}}_{T}(\Gamma)= & \sup _{z}\left\|\left|\nabla_{x}\right|^{\frac{1}{2}+\epsilon} \Gamma(t, x+z, x)\right\|_{L^{2}\left([0, T] \times \mathbb{R}^{3}\right)} \\
& +\left.\sup _{z}\|\| \nabla_{x}\right|^{1 / 2} \Gamma(t, x+z, x) \|_{L^{2}\left([0, T] \times \mathbb{R}^{3}\right)} \\
& +\left\|<\nabla_{x}>^{\frac{1}{2}+\epsilon}<\nabla_{y}>^{\frac{1}{2}+\epsilon} \Gamma(t, x, y)\right\|_{L^{\infty}\left([0, T] \times L^{2}\left(\mathbb{R}^{6}\right)\right)} \\
& \mathbf{N}_{T}(\phi)=\left\|<\nabla_{x}>^{\frac{1}{2}+\epsilon} \phi\right\|_{L^{\infty}([0, T]) L^{2}}+\left\|<\nabla_{x}>^{\frac{1}{2}+\epsilon} \phi\right\|_{L^{2}([0, T]) L^{6}}
\end{aligned}
$$

Based on energy and Strichartz estimates, the collapsing estimates of Lemma (5.1) and general properties of $X$ spaces which allow one to transfer estimates for solutions to the homogeneous equation to estimates in $X^{1 / 2+\delta}$ spaces we have

$$
\begin{aligned}
& \mathbf{N}_{T}(\Lambda) \lesssim\left\|<\nabla_{x}>^{\frac{1}{2}+\epsilon}<\nabla_{y}>^{\frac{1}{2}+\epsilon} \Lambda\right\|_{X_{S}^{\frac{1}{2}+\delta}} \\
& \dot{\mathbf{N}}_{T}(\Gamma) \lesssim\left\|<\nabla_{x}>^{\frac{1}{2}+\epsilon}<\nabla_{y}>^{\frac{1}{2}+\epsilon} \bar{\Gamma}\right\|_{X_{W}^{\frac{1}{2}+\delta}} \\
& \mathbf{N}_{T}(\phi \otimes \phi)+\dot{\mathbf{N}}_{T}(\phi \otimes \bar{\phi}) \lesssim\left\|<\nabla_{x}>^{\frac{1}{2}+\epsilon} \phi\right\|_{X^{\frac{1}{2}+\delta}}^{2}
\end{aligned}
$$

Our basic estimates for the inhomogeneous linear equations are

Proposition 5.8. If $\Gamma$ and $\phi$ satisfy

$$
\begin{aligned}
& \mathbf{S}_{ \pm} \Gamma=F \\
& \mathbf{S} \phi=G
\end{aligned}
$$

$0 \leq T \leq 1$, and $2-<2,6 / 5+>6 / 5$ are fixed numbers which can be chosen close to 2 and $6 / 5$, then

$$
\begin{aligned}
& \dot{\mathbf{N}}_{T}(\Gamma) \\
& \lesssim\left\|<\nabla_{x}>^{\frac{1}{2}+\epsilon}<\nabla_{y}>^{\frac{1}{2}+\epsilon} \Gamma(0, x, y)\right\|_{L^{2}(d x d y)} \\
& \quad+\left\|<\nabla_{x}>^{\frac{1}{2}+\epsilon}<\nabla_{y}>^{\frac{1}{2}+\epsilon} F\right\|_{L^{2-}[0, T] L^{6 / 5+}(d x) L^{2}(d y)} \\
& \mathbf{N}_{T}(\phi) \\
& \lesssim\left\|<\nabla_{x}>^{\frac{1}{2}+\epsilon} \phi(0, \cdot)\right\|_{L^{2}(d x)}+\left\|<\nabla_{x}>^{\frac{1}{2}+\epsilon} G\right\|_{L^{2-}[0, T] L^{6 / 5+}(d x)} \\
& \mathbf{N}_{T}(\phi \otimes \phi)+\dot{\mathbf{N}}_{T}(\phi \otimes \bar{\phi}) \lesssim \mathbf{N}_{T}(\phi)^{2}
\end{aligned}
$$

Similar estimates hold when $x$ and $y$ are reversed. 
Proof. We will prove this for the equation for $\Gamma$, the other one for $\phi$ being easier. The standard energy estimate and the collapsing estimate of lemma (5.1) prove that if $\mathbf{S}_{ \pm} \Gamma=0$, then

$$
\dot{\mathbf{N}}_{T}(\Gamma) \lesssim\left\|<\nabla_{x}>^{\frac{1}{2}+\epsilon}<\nabla_{y}>^{\frac{1}{2}+\epsilon} \Gamma_{0}(x, y)\right\|_{L^{2}(d x d y)}
$$

From here and general properties of $X^{1 / 2+\delta}$ spaces, we get, for any $\delta>0$,

$$
\dot{\mathbf{N}}_{T}(\Gamma) \lesssim_{\delta}\left\|<\nabla_{x}>^{\frac{1}{2}+\epsilon}<\nabla_{y}>^{\frac{1}{2}+\epsilon} \Gamma\right\|_{X_{W}^{1 / 2+\delta}}
$$

uniformly in $T$.

Finally, to prove the stated estimate, let $F_{T}=F$ in $[0, T]$, and 0 otherwise. Let $\Gamma_{T}$ be the solution to

$$
\begin{aligned}
& \mathbf{S}_{ \pm} \Gamma_{T}=F_{T} \\
& \Gamma_{T}(0, \cdot)=\Gamma(0, \cdot)
\end{aligned}
$$

Then $\Gamma=\Gamma_{T}$ in $[0, T]$ and recalling $0<T \leq 1$ and $\chi=1$ on $[0,1]$,

$$
\begin{aligned}
& \dot{\mathbf{N}}_{T}(\Gamma)=\dot{\mathbf{N}}_{T}\left(\chi(t) \Gamma_{T}\right) \\
& \lesssim\left\|<\nabla_{x}>^{\frac{1}{2}+\epsilon}<\nabla_{y}>^{\frac{1}{2}+\epsilon} \chi(t) \Gamma_{T}\right\|_{X_{S}^{\frac{1}{2}+\delta}} \\
& \lesssim\left\|<\nabla_{x}>^{\frac{1}{2}+\epsilon}<\nabla_{y}>^{\frac{1}{2}+\epsilon} \Gamma_{0}(x, y)\right\|_{L^{2}(d x d y)} \\
& +\left\|<\nabla_{x}>^{\frac{1}{2}+\epsilon}<\nabla_{y}>^{\frac{1}{2}+\epsilon} F_{T}\right\|_{\left.X_{S}^{-\frac{1}{2}+\delta} \quad(\text { by (151) })\right)} \\
& \lesssim\left\|<\nabla_{x}>^{\frac{1}{2}+\epsilon}<\nabla_{y}>^{\frac{1}{2}+\epsilon} \Gamma_{0}(x, y)\right\|_{L^{2}(d x d y)} \\
& +\left\|<\nabla_{x}>^{\frac{1}{2}+\epsilon}<\nabla_{y}>^{\frac{1}{2}+\epsilon} F_{T}\right\|_{L^{2-}(d t) L^{6 / 5+}(d x) L^{2}(d y)} \text { (by ([64)) } \\
& =\left\|<\nabla_{x}>^{\frac{1}{2}+\epsilon}<\nabla_{y}>^{\frac{1}{2}+\epsilon} \Gamma_{0}(x, y)\right\|_{L^{2}(d x d y)} \\
& +\left\|<\nabla_{x}>^{\frac{1}{2}+\epsilon}<\nabla_{y}>^{\frac{1}{2}+\epsilon} F\right\|_{L^{2-}[0, T] L^{6 / 5+}(d x) L^{2}(d y)}
\end{aligned}
$$

The same type of result holds for the equation for $\Lambda$.

Proposition 5.9. There exist numbers $2-<2,6 / 5+>6 / 5$, which can be chosen arbitrarily close to 2 and 6/5 such that, if $\Lambda$ satisfies

$$
\begin{aligned}
& \left(\mathbf{S}+\frac{1}{N} v_{N}(x-y)\right) \Lambda=F \\
& \Lambda(0, \cdot)=\Lambda_{0}(\cdot)
\end{aligned}
$$

$\beta<2 / 3$, and $0 \leq T \leq 1$, then 


$$
\begin{aligned}
& \mathbf{N}_{T}(\Lambda) \\
& \lesssim\left\|<\nabla_{x}>^{\frac{1}{2}+\epsilon}<\nabla_{y}>^{\frac{1}{2}+\epsilon} \Lambda_{0}(x, y)\right\|_{L^{2}(d x d y)} \\
& +\left\|<\nabla_{x}>^{\frac{1}{2}+\epsilon}<\nabla_{y}>^{\frac{1}{2}+\epsilon} F\right\|_{L^{2-}[0, T] L^{6 / 5+}(d x) L^{2}(d y)}
\end{aligned}
$$

Similar estimates hold for $\left(\frac{\partial}{\partial t}\right)^{i} \nabla_{x+y}^{j} \Lambda$ :

$$
\begin{aligned}
& \mathbf{N}_{T}\left(\left(\frac{\partial}{\partial t}\right)^{i} \Lambda\right) \\
& \lesssim\left\|<\nabla_{x}>^{\frac{1}{2}+\epsilon}<\nabla_{y}>\left.^{\frac{1}{2}+\epsilon}\left(\frac{\partial}{\partial t}\right)^{i} \nabla_{x+y}^{j} \Lambda(t, x, y)\right|_{t=0}\right\|_{L^{2}(d x d y)} \\
& +\left\|<\nabla_{x}>^{\frac{1}{2}+\epsilon}<\nabla_{y}>^{\frac{1}{2}+\epsilon}\left(\frac{\partial}{\partial t}\right)^{i} \nabla_{x+y}^{j} F\right\|_{L^{2-}[0, T] L^{6 / 5+}(d x) L^{2}(d y)}
\end{aligned}
$$

Proof. The proof is the same as the one of Proposition (5.8), except that, because of the potential $v_{N}$ we don't get the estimate (51) from general principles but use Proposition (5.7). To prove (77), first differentiate the equation, noting that these derivatives commute with the potential, then apply the previous result.

\section{THE NON-LINEAR EQUATIONS}

Now we come to our main PDE result

Theorem 6.1. Let $\Lambda, \Gamma$ and $\phi$ be solutions of (33b), (33c), (33a) with initial conditions $\phi_{0}, k_{0} \in \mathcal{S}$. There exists $N_{0}$ such that for all $N \geq N_{0}$, the following estimates hold:

$$
\begin{gathered}
\mathbf{N}_{T}(\Lambda) \lesssim\left\|<\nabla_{x}>^{\frac{1}{2}+\epsilon}<\nabla_{y}>^{\frac{1}{2}+\epsilon} \Lambda(0, \cdot)\right\|_{L^{2}} \\
+T^{\text {smallpower }}\left(\mathbf{N}_{T}(\Lambda) \dot{\mathbf{N}}_{T}(\Gamma)+\mathbf{N}_{T}^{4}(\phi)\right) \\
\dot{\mathbf{N}}_{T}(\Gamma) \lesssim\left\|<\nabla_{x}>^{\frac{1}{2}+\epsilon}<\nabla_{y}>^{\frac{1}{2}+\epsilon} \Gamma(0, \cdot)\right\|_{L^{2}} \\
+T^{\text {smallpower }}\left(\mathbf{N}_{T}^{2}(\Lambda)+\dot{\mathbf{N}}_{T}^{2}(\Gamma)+\mathbf{N}_{T}^{4}(\phi)\right) \\
\mathbf{N}_{T}(\phi) \lesssim\left\|<\nabla_{x}>^{\frac{1}{2}+\epsilon} \phi(0, \cdot)\right\|_{L^{2}} \\
+T^{\text {smallpower }}\left(\mathbf{N}_{T}(\Lambda)+\dot{\mathbf{N}}_{T}(\Gamma)+\mathbf{N}_{T}^{2}(\phi)\right) \mathbf{N}_{T}(\phi)
\end{gathered}
$$


So there exists $T_{0}>0$ such that, if $T \leq T_{0}$,

$$
\begin{aligned}
& \mathbf{N}_{T}(\Lambda)+\dot{\mathbf{N}}_{T}(\Gamma)+\mathbf{N}_{T}(\phi) \\
& \lesssim\left\|<\nabla_{x}>^{\frac{1}{2}+\epsilon}<\nabla_{y}>^{\frac{1}{2}+\epsilon} \Lambda(0, \cdot)\right\|_{L^{2}}+\left\|<\nabla_{x}>^{\frac{1}{2}+\epsilon}<\nabla_{y}>^{\frac{1}{2}+\epsilon} \Gamma(0, \cdot)\right\|_{L^{2}} \\
& +\left\|<\nabla_{x}>^{\frac{1}{2}+\epsilon} \phi(0, \cdot)\right\|_{L^{2}}
\end{aligned}
$$

Also, similar estimates hold for the derivatives which commute with the potential:

$$
\begin{aligned}
& \mathbf{N}_{T}\left(\frac{\partial}{\partial t} \nabla_{x+y}^{j} \Lambda\right)+\dot{\mathbf{N}}_{T}\left(\frac{\partial}{\partial t} \nabla_{x+y}^{j} \Gamma\right)+\mathbf{N}_{T}\left(\frac{\partial}{\partial t} \nabla_{x}^{j} \phi\right) \\
& \lesssim\left\|<\nabla_{x}>^{\frac{1}{2}+\epsilon}<\nabla_{y}>\left.^{\frac{1}{2}+\epsilon} \frac{\partial}{\partial t} \nabla_{x+y}^{j} \Lambda\right|_{t=0}\right\|_{L^{2}} \\
& +\left\|<\nabla_{x}>^{\frac{1}{2}+\epsilon}<\nabla_{y}>\left.^{\frac{1}{2}+\epsilon} \frac{\partial}{\partial t} \nabla_{x+y}^{j} \Gamma\right|_{t=0}\right\|_{L^{2}} \\
& +\left\|<\nabla_{x}>\left.^{\frac{1}{2}+\epsilon} \frac{\partial}{\partial t} \nabla_{x}^{j} \phi\right|_{t=0}\right\|_{L^{2}}
\end{aligned}
$$

The time interval $T_{0}$ and the implicit constants in the above inequalities depend only on

$$
\begin{aligned}
& \left\|<\nabla_{x}>^{\frac{1}{2}+\epsilon}<\nabla_{y}>^{\frac{1}{2}+\epsilon} \Lambda(0, \cdot)\right\|_{L^{2}}+ \\
& \left\|<\nabla_{x}>^{\frac{1}{2}+\epsilon}<\nabla_{y}>^{\frac{1}{2}+\epsilon} \Gamma(0, \cdot)\right\|_{L^{2}} \\
& \left\|<\nabla_{x}>^{\frac{1}{2}+\epsilon} \phi(0, \cdot)\right\|_{L^{2}}
\end{aligned}
$$

Proof. For equation (33a), which can be abbreviated as

$$
\begin{aligned}
& \frac{1}{i} \partial_{t} \phi-\Delta \phi \\
& =-\left(v_{N} \Lambda\right) \circ \bar{\phi}-\left(v_{N} \bar{\Gamma}\right) \circ \phi-\left(v_{N} * \operatorname{Tr} \Gamma\right) \cdot \phi+2\left(v_{N} *|\phi|^{2}\right) \phi \\
& :=\operatorname{RHS}(33 \mathrm{a})
\end{aligned}
$$

we first apply Proposition (5.8):

$$
\begin{aligned}
& \mathbf{N}_{T}(\phi) \lesssim\left\|<\nabla_{x}>^{\frac{1}{2}+\epsilon} \phi(0, \cdot)\right\|_{L^{2}}+\|<\nabla_{x}>^{\frac{1}{2}+\epsilon} R H S(\text { (33a) }) \|_{L^{2-}([0, T]) L^{6 / 5+}(d x)} \\
& \lesssim\left\|<\nabla_{x}>^{\frac{1}{2}+\epsilon} \phi(0, \cdot)\right\|_{L^{2}}+T^{\text {small power }}\left\|<\nabla_{x}>^{\frac{1}{2}+\epsilon} R H S(\underline{33 \mathrm{a}})\right\|_{L^{2}([0, T]) L^{6 / 5+}(d x)} \\
& \lesssim\left\|<\nabla_{x}>^{\frac{1}{2}+\epsilon} \phi(0, \cdot)\right\|_{L^{2}}+T^{\text {small power }}\left(\mathbf{N}_{T}(\Lambda)+\dot{\mathbf{N}}_{T}(\Gamma)+\mathbf{N}_{T}^{2}(\phi)\right) \mathbf{N}_{T}(\phi)
\end{aligned}
$$

The last line follow from the classical fractional Leibnitz rule in $L^{p}$ spaces due to Kato and Ponce, [26]. We only present a typical term 
in RHS(33a):

$$
\begin{aligned}
& \left\|<\nabla_{x}>^{\frac{1}{2}+\epsilon}\left(\left(v_{N} * \operatorname{Tr} \Gamma\right) \cdot \phi\right)\right\|_{L^{2}([0, T]) L^{6 / 5+}(d x)} \\
& \lesssim\left\|\left(v_{N} * \operatorname{Tr} \Gamma\right) \cdot \phi\right\|_{L^{2}([0, T]) L^{6 / 5+}(d x)}+\left\|\left|\nabla_{x}\right|^{\frac{1}{2}+\epsilon}\left(\left(v_{N} * \operatorname{Tr} \Gamma\right) \cdot \phi\right)\right\|_{L^{2}([0, T]) L^{6 / 5+}(d x)} \\
& \lesssim\|\operatorname{Tr} \Gamma\|_{L^{2}([0, T]) L^{3}(d x)}\|\phi\|_{L^{\infty} L^{2+}} \\
& +\left.\left.\|\| \nabla_{x}\right|^{\frac{1}{2}+\epsilon} \operatorname{Tr} \Gamma\left\|_{L^{2}([0, T]) L^{2}(d x)}\right\| \phi\left\|_{L^{\infty} L^{3+}}+\right\| \operatorname{Tr} \Gamma\left\|_{L^{2}([0, T]) L^{3+}(d x)}\right\| \nabla_{x}\right|^{\frac{1}{2}+\epsilon} \phi \|_{L^{\infty} L^{2}} \\
& \lesssim \dot{\mathbf{N}}_{T}(\Gamma) \mathbf{N}_{T}(\phi)
\end{aligned}
$$

Now we deal with the equation (33b), which can be abbreviated as

$$
\begin{aligned}
& \left(\mathbf{S}+\frac{1}{N} v_{N}\right) \Lambda \\
& =-\left(v_{N} \Lambda\right) \circ \Gamma-\bar{\Gamma} \circ\left(v_{N} \Lambda\right) \\
& -\left(\left(v_{N} * \operatorname{Tr} \Gamma\right)(x)+\left(v_{N} * \operatorname{Tr} \Gamma\right)(y)\right) \Lambda(x, y) \\
& -\left(v_{N} \bar{\Gamma}\right) \circ \Lambda-\Lambda \circ\left(v_{N} \Gamma\right) \\
& +2\left(v_{N} *|\phi|^{2}\right)(x) \phi(x) \phi(y)+2\left(v_{N} *|\phi|^{2}\right)(y) \phi(y) \phi(x) \\
& :=\operatorname{RHS}(\underline{33 \mathrm{~b}})
\end{aligned}
$$

Applying Proposition (5.9) to the equation (33b)

$$
\begin{aligned}
& \mathbf{N}_{T}(\Lambda) \lesssim\left\|<\nabla_{x}>^{\frac{1}{2}+\epsilon}<\nabla_{y}>^{\frac{1}{2}+\epsilon} \Lambda(0, \cdot)\right\|_{L^{2}} \\
& +\left\|<\nabla_{x}>^{\frac{1}{2}+\epsilon}<\nabla_{y}^{\frac{1}{2}+\epsilon}>\operatorname{RHS}(\underline{33 \mathrm{~b}})\right\|_{L^{2-}[0, T] L^{6 / 5+}(d x) L^{2}(d y)}
\end{aligned}
$$

We would like to estimate $<\nabla_{x}>^{\frac{1}{2}+\epsilon}<\nabla_{y}>^{\frac{1}{2}+\epsilon} R H S(\underline{33 \mathrm{~b}})$ in $L^{2} L^{\frac{6}{5}+} L^{2}$ to gain a small power of $T$ from Cauchy-Schwarz in time.

Typical term in RHS (33b):

$$
\begin{aligned}
& <\nabla_{x}>^{\frac{1}{2}+\epsilon}<\nabla_{y}^{\frac{1}{2}+\epsilon}>\left(\left(v_{N} \Lambda\right) \circ \Gamma(x, y)\right) \\
& =\int v_{N}(z)<\nabla_{x}>^{\frac{1}{2}+\epsilon}<\nabla_{y}^{\frac{1}{2}+\epsilon}>(\Lambda(x, x-z) \Gamma(x-z, y)) d z
\end{aligned}
$$


Applying the fractional Leibniz rule in $L^{p}$ spaces,

$$
\begin{aligned}
& \int v_{N}(z) \|<\nabla_{x}>^{\frac{1}{2}+\epsilon}<\nabla_{y}^{\frac{1}{2}+\epsilon}>(\Lambda(x, x-z) \Gamma(x-z, y)) \|_{L^{2}([0, T]) L^{\frac{6}{5}}(d x) L^{2}(d y)} d z \\
& \lesssim \int v_{N}(z) \|<\nabla_{x}>^{\frac{1}{2}+\epsilon} \Lambda(x, x-z) \|_{L^{2}([0, T]) L^{2}(d x)} \times \\
& \|<\nabla_{y}^{\frac{1}{2}+\epsilon}>\Gamma(x-z, y) \|_{L^{\infty}([0, T]) L^{3+}(d x) L^{2}(d y)} d z \\
&+\int v_{N}(z)\|\Lambda(x, x-z)\|_{L^{2}([0, T]) L^{3+}(d x)} \times \\
&\left\|<\nabla_{x}>^{\frac{1}{2}+\epsilon}<\nabla_{y}^{\frac{1}{2}+\epsilon}>\Gamma(x-z, y)\right\|_{L^{\infty}([0, T]) L^{2}(d x) L^{2}(d y)} d z \\
& \lesssim \mathbf{N}_{T}(\Lambda) \dot{\mathbf{N}}_{T}(\Gamma)
\end{aligned}
$$

All other terms are treated in a similar manner. In fact, all terms on the RHS(33b) and RHS( $(\underline{33 \mathrm{C}})$ are of the form $\left(v_{N} F\right) \circ G$ or $\left(v_{N} * \operatorname{TrF}\right) G$ where $F, G$ can be $\Lambda, \Gamma, \phi \otimes \phi$ of $\phi \otimes \bar{\phi}$ (or their complex conjugates) and $\mathbf{N}_{T}(F), \mathbf{N}_{T}(G)$ are estimated as above by Proposition (5.8) or Proposition (5.9).

\section{Estimates FOR $\operatorname{sh}(2 k)$}

Recall the equation (24a), which can be written explicitly as

$$
\begin{aligned}
& \mathbf{S}(\operatorname{sh}(2 k))=-2 v_{N} \Lambda \\
& -\left(v_{N} \Lambda\right) \circ p_{2}-\bar{p}_{2} \circ\left(v_{N} \Lambda\right) \\
& -\left(\left(v_{N} * \operatorname{Tr} \Gamma\right)(x)+\left(v_{N} * \operatorname{Tr} \Gamma\right)(y)\right) \operatorname{sh}(2 k)(x, y)-\left(v_{N} \Gamma\right) \circ \operatorname{sh}(2 k)-\operatorname{sh}(2 k) \circ\left(v_{N} \Gamma\right) \\
& :=\operatorname{RHS}(79)
\end{aligned}
$$

Now that we control the quantities (78) we use proofs similar to those of section 4 of [23], or section 3 of [33] with $-v_{N} \Lambda$ playing the role of $m$ and $\tilde{\mathbf{S}}$ playing the role of $\mathbf{S}$, at least locally in time. The crucial ingredient is that

$$
\begin{aligned}
& \sup _{z}\|\Lambda(t, x+z, x)\|_{L^{2}(d x)}+\sup _{z}\left\|\left(\frac{\partial}{\partial t}\right)^{i} \Lambda(t, x+z, x)\right\|_{L^{2}(d x)} \\
& +\|\Lambda\|_{L^{\infty}}+\left\|\left(\frac{\partial}{\partial t}\right)^{i} \Lambda\right\|_{L^{\infty}} \lesssim 1
\end{aligned}
$$

for $i=0,1$. The $\nabla_{x+y}$ derivatives have been used to control $\|\Lambda(t, x+z, x)\|_{L^{\infty}(d x)} \leq C \sum_{j \leq 2}\left\|\left(\nabla_{x+y}^{j} \Lambda\right)(t, x+z, x)\right\|_{L^{2}(d x)}$. 
The reader is warned, with apologies, that $\mathbf{S}$ in [23] is not the $\mathbf{S}$ of the current paper (which is a purely differential operator), but what was called $\mathbf{S}_{\text {old }}$ in the introduction.

In this section, we will prove

Theorem 7.1. Let $\operatorname{sh}(2 k), \operatorname{ch}(2 k)$ satisfy the equations (24a), (24b) with initial conditions as in Theorem (2.5). Then, for $T_{0}$ as in Theorem (6.1) and $0 \leq j \leq 2$,

$$
\begin{aligned}
& \left\|\nabla_{x+y}^{j} \operatorname{sh}(2 k)(t, \cdot, \cdot)\right\|_{L^{2}(d x d y)} \lesssim 1 \\
& \sup _{x}\|\operatorname{sh}(2 k)(t, x, \cdot)\|_{L^{2}(d y)} \lesssim 1 \quad\left(0 \leq t \leq T_{0}\right)
\end{aligned}
$$

Define $\operatorname{ch}(k)=\delta(x-y)+p$. The following is immediate, as in the proof of Corollary 4.2 in [23]:

Corollary 7.2. The following estimates hold uniformly in $0 \leq t \leq T_{0}$

$$
\begin{aligned}
& \|\operatorname{sh}(k)(t, \cdot, \cdot)\|_{L^{2}(d x d y)} \lesssim 1 \\
& \|p(t, \cdot, \cdot)\|_{L^{2}(d x d y)} \lesssim 1 \\
& \sup _{x}\|\operatorname{sh}(k)(t, x, \cdot)\|_{L^{2}(d y)} \lesssim 1 \\
& \sup _{x}\|p(t, x, \cdot)\|_{L^{2}(d y)} \lesssim 1
\end{aligned}
$$

We start with some preliminary lemmas.

Lemma 7.3. (replacing Lemma 4.4 in [23].) Let $s_{a}^{0}$ be the solution to

$$
\begin{array}{r}
\mathbf{S} s_{a}^{0}=-2 v_{N}(x-y) \Lambda \\
s_{a}^{0}(0, x, y)=\operatorname{sh}(2 k)(0, x, y)
\end{array}
$$

Then

$$
\begin{aligned}
& \left\|s_{a}^{0}(t, \cdot, \cdot)\right\|_{L^{2}(d x d y)} \lesssim 1 \\
& \left(0 \leq t \leq T_{0}\right)
\end{aligned}
$$

with similar estimates for $\nabla_{x+y}^{j} s_{a}^{0}$.

This lemma is a particular case (with $F=v_{N}(x-y) \Lambda(t, x, y)$ of a more general result:

Lemma 7.4. Let $F$ be a function of $6+1$ variables $\left(k \geq 0, x, y \in \mathbb{R}^{3}\right.$, $t \in[0,1])$ and let

$$
E(x, y, t)=\int_{0}^{t} e^{i(t-s) \Delta_{x, y}} F(s) d s
$$


Then

$$
\begin{aligned}
& \|E(t, \cdot)\|_{L^{2}} \\
& \lesssim \sup _{0 \leq s \leq t}\left(\|F(s, \cdot)\|_{L^{2}(d(x+y)) L^{1}(d(x-y))}+\left\|\frac{\partial}{\partial s} F(s, \cdot)\right\|_{L^{2}(d(x+y)) L^{1}(d(x-y))}\right)
\end{aligned}
$$

Proof. Change variables, so we work in $x, y$ rather than $x-y, x+y$ coordinates. In these coordinates, integrating by parts,

$$
\begin{aligned}
& E(t, \cdot)=\int_{0}^{t} e^{i(t-s) \Delta_{x, y}} F(s, \cdot) d s \\
& =\int_{0}^{t} e^{i(t-s) \Delta_{x, y}} \Delta_{x, y}^{-1} \frac{\partial}{\partial s} F(s, \cdot) d s \\
& +e^{i t \Delta_{x, y}} \Delta_{x, y}^{-1} F(0, \cdot)-\Delta^{-1} F(t, \cdot)
\end{aligned}
$$

We are going to project in frequencies dual to $x$ only. For the low frequency case we use (84):

$$
\begin{aligned}
& \left\|P_{|\xi| \leq 1} E(t, \cdot)\right\|_{L^{2}}=\left\|\int_{0}^{t} e^{i(t-s) \Delta_{x, y}} P_{|\xi| \leq 1} F(s, \cdot) d s\right\|_{L^{2}} \\
& \leq \sup _{0 \leq s \leq t}\left\|P_{|\xi| \leq 1} F(s, \cdot)\right\|_{L^{2}(d x d y)} \\
& \lesssim \sup _{0 \leq s \leq t}\|F(s, \cdot)\|_{L^{2}(d y) L^{1}(d x)}
\end{aligned}
$$

where we have used the fact that the (physical space) kernel corresponding to $P_{|\xi| \leq 1}$ is in $L^{2}(d x)$. For the high frequency case we use (85). We only write down one term, the boundary terms being similar:

$$
\begin{aligned}
& \left\|P_{|\xi| \geq 1} E(t, \cdot)\right\|_{L^{2}}=\left\|\int_{0}^{t} e^{i(t-s) \Delta_{x, y}} P_{|\xi| \geq 1} \Delta_{x, y}^{-1} \frac{\partial}{\partial s} F(s, \cdot) d s\right\|_{L^{2}} \\
& \leq \sup _{0 \leq s \leq t}\left\|P_{|\xi| \geq 1} \Delta_{x}^{-1} \frac{\partial}{\partial s} F(s, \cdot) d s\right\|_{L^{2}(d x d y)} \\
& \lesssim \sup _{0 \leq s \leq t}\left\|\frac{\partial}{\partial s} F(s, \cdot)\right\|_{L^{2}(d y) L^{1}(d x)}
\end{aligned}
$$

where we have used that the kernel of $P_{|\xi| \geq 1} \Delta_{x}^{-1}$ in in $L^{2}(d x)$.

Next, we include the potential terms:

Lemma 7.5. Let $s_{a}$ be the solution to

$$
\begin{aligned}
& \tilde{\mathbf{S}} s_{a}=-2 v_{N} \Lambda \\
& s_{a}(0, x, y)=\operatorname{sh}(2 k)(0, x, y)
\end{aligned}
$$




$$
\left\|s_{a}^{0}(t, \cdot, \cdot)\right\|_{L^{2}(d x d y)} \lesssim 1\left(0 \leq t \leq T_{0}\right)
$$

with similar estimates for $\nabla_{x+y}^{j} s_{a}$.

Proof. Let $V$ be the "potential" part of $\tilde{S}$ :

$$
V(u)=\left(\left(v_{N} * \operatorname{Tr} \Gamma\right)(x)+\left(v_{N} * \operatorname{Tr} \Gamma\right)(y)\right) u+\left(v_{N} \Gamma\right) \circ u+u \circ\left(v_{N} \Gamma\right)
$$

Form the estimates (78) for $\Gamma$ we see that $\left|\nabla_{x+y}^{j} \Gamma\right| \lesssim 1$, thus $V$ and $\left[V, \nabla_{x+y}^{j}\right]$ have bounded operator norm (on $\left.L^{2}\right)$. Write $s_{a}=s_{a}^{0}+s_{a}^{1}\left(s_{a}^{0}\right.$ as in the previous lemma) so that $s_{a}^{1}$ satisfies the equation

$$
\begin{gathered}
\tilde{\mathbf{S}} s_{a}^{1}=-V\left(s_{a}^{0}\right) \\
s_{a}^{1}(0, x, y)=0
\end{gathered}
$$

Using energy estimates and the previous lemma we see

$$
\left\|\nabla_{x+y}^{j} s_{a}^{1}\right\|_{L^{2}} \lesssim 1
$$

The result follows from the previous lemma.

Finally, we can prove Theorem (7.1).

Proof. (of theorem (7.1)) Write $\operatorname{sh}(2 k):=s_{2}=s_{a}+s_{e}$ where $s_{a}$ satisfies (86) and $\operatorname{ch}(2 k)=\delta(x-y)+p_{2}$. In analogy with (64a), (64b) of [23], they satisfy

$$
\begin{array}{r}
\tilde{\mathbf{S}}\left(s_{e}\right)=-\left(v_{N} \Lambda\right) \circ p_{2}-\bar{p}_{2} \circ\left(v_{N} \Lambda\right) \\
\tilde{\mathbf{W}}\left(\bar{p}_{2}\right)=-\left(v_{N} \Lambda\right) \circ \bar{s}_{a}+s_{a} \circ\left(v_{N} \Lambda\right) \\
-\left(v_{N} \Lambda\right) \circ \bar{s}_{e}+s_{e} \circ\left(v_{N} \Lambda\right) \\
:=M-\left(v_{N} \Lambda\right) \circ \bar{s}_{e}+s_{e} \circ\left(v_{N} \Lambda\right)
\end{array}
$$

Using the result of Lemma (7.5), as well as estimates (78) for $\Lambda$ we see that

$$
\left\|\nabla_{x+y}^{j} M\right\|_{L^{2}(d x d y)} \lesssim 1 \quad\left(0 \leq t \leq T_{0}\right)
$$

and the result follows by energy estimates. 
Finally, to prove (82) we will use the $L^{2}$ estimate (81):

$$
\begin{aligned}
& \|\| \operatorname{sh}(2 k)(x, z)\left\|_{L^{2}(d z)}\right\|_{L^{\infty}(d x)}=\|\| \operatorname{sh}(2 k)(x, x+z)\left\|_{L^{2}(d z)}\right\|_{L^{\infty}(d x)} \\
& \leq\|\| \operatorname{sh}(2 k)(x, x+z)\left\|_{L^{\infty}(d x)}\right\|_{L^{2}(d z)} \leq C \sum_{j=0}^{2}\|\| \nabla_{x}^{j}(\operatorname{sh}(2 k)(x, x+z))\left\|_{L^{2}(d x)}\right\|_{L^{2}(d z)} \\
& =C \sum_{j=0}^{2}\|\|\left(\nabla_{x+y}^{j} \operatorname{sh}(2 k)\right)(x, x+z)\left\|_{L^{2}(d x)}\right\|_{L^{2}(d z)} \\
& =C \sum_{j=0}^{2}\left\|\left(\nabla_{x+y}^{j} \operatorname{sh}(2 k)\right)(x, y)\right\|_{L^{2}(d x d y)} \leq C
\end{aligned}
$$

\section{The Reduced Hamiltonian}

Recall (11) for the definition of the reduced Hamiltonian. $\mathcal{H}_{\text {red }}$ was computed, for instance, in Section 5 of [23]. We will write it in a different way, using Wick's theorem. Recall the conjugation formulas

$$
\begin{aligned}
e^{\mathcal{B}} a_{x} e^{-\mathcal{B}} & =\int\left(\operatorname{ch}(k)(y, x) a_{y}+\operatorname{sh}(k)(y, x) a_{y}^{*}\right) d y \\
& =a(\overline{\operatorname{ch}(k)}(x, \cdot))+a^{*}(\operatorname{sh}(k)(x, \cdot)):=b_{x} \\
e^{\mathcal{B}} a_{x}^{*} e^{-\mathcal{B}} & =\int\left(\overline{\operatorname{sh}(k)}(y, x) a_{y}+\overline{\operatorname{ch}(k)}(y, x) a_{y}^{*}\right) d y \\
& =a(\overline{\operatorname{sh}(k)}(x, \cdot))+a^{*}(\operatorname{ch}(k)(x, \cdot)):=b_{x}^{*} .
\end{aligned}
$$


The reduced Hamiltonian is

$$
\begin{aligned}
& \mathcal{H}_{\text {red }}=N \mu_{0}(t) \\
& +N^{1 / 2} \int d x\left\{h(\phi(t, x)) b_{x}^{*}+\bar{h}(\phi(t, x)) b_{x}\right\} \\
& +\frac{1}{i} \frac{\partial}{\partial t}\left(e^{\mathcal{B}(k(t))}\right) e^{-\mathcal{B}(k(t)}+\int d x b_{x}^{*} \Delta b_{x} \\
& -\frac{1}{2} \int d x d y v_{N}(x-y)\left(\bar{\phi}(x) \bar{\phi}(y) b_{x} b_{y}+\phi(x) \phi(y) b_{x}^{*} b_{y}^{*}+2 \phi(x) \bar{\phi}(y) b_{x}^{*} b_{y}\right) \\
& -\int d x\left(v_{N} *|\phi|^{2}\right) b_{x}^{*} b_{x} \\
& -\frac{1}{\sqrt{N}} \int d x_{1} d x_{2}\left\{v_{N}\left(x_{1}-x_{2}\right)\left(\bar{\phi}\left(x_{2}\right) b_{x_{1}}^{*} b_{x_{1}} b_{x_{2}}+\phi\left(x_{2}\right) b_{x_{1}}^{*} b_{x_{2}}^{*} b_{x_{1}}\right)\right\} \\
& -\frac{1}{2 N} \int d x_{1} d x_{2}\left\{v_{N}\left(x_{1}-x_{2}\right) b_{x_{1}}^{*} b_{x_{2}}^{*} b_{x_{1}} b_{x_{2}}\right\}
\end{aligned}
$$

The functions $\mu_{0}(t)$ and $h(\phi(t, x))$ appearing in (88a), (88b) are given below,

$$
\begin{aligned}
& \mu_{0}:=\int d x\left\{\frac{1}{2 i}\left(\phi \bar{\phi}_{t}-\bar{\phi} \phi_{t}\right)-|\nabla \phi|^{2}\right\}-\frac{1}{2} \int d x d y\left\{v_{N}(x-y)|\phi(x)|^{2}|\phi(y)|^{2}\right\} \\
& h(\phi(t, x)):=-\frac{1}{i} \partial_{t} \phi+\Delta \phi-\left(v_{N} *|\phi|^{2}\right) \phi .
\end{aligned}
$$

We re-arrange the terms in $\mathcal{H}_{\text {red }}$ using Wick's theorem . Define the contraction of $A(f):=a\left(\bar{f}_{1}\right)+a^{*}\left(f_{2}\right)$ and $A(g):=a\left(\bar{g}_{1}\right)+a^{*}\left(g_{2}\right)$ to be $C(A(f), A(g))=\left[a\left(\bar{f}_{1}\right), a^{*}\left(g_{2}\right)\right]=\int \bar{f}_{1} g_{2}$, and define the normal order $\operatorname{Nor}(A(f) A(g) A(h) A(k))$ to be $A(f) A(g) A(h) A(k)$ expanded and rearranged so all starred terms have been moved to the left, as if they commuted with unstarred terms. Wick's theorem, which can easily be proved by induction, says, in particular, that

$$
\begin{aligned}
& A(g) A(h) A(k)=\operatorname{Nor}(A(g) A(h) A(k)) \\
& +C(A(g), A(h)) A(k)+C(A(g), A(k)) A(h)+C(A(h), A(k)) A(g)
\end{aligned}
$$




$$
\begin{aligned}
& A(f) A(g) A(h) A(k) \\
& =\operatorname{Nor}(A(f) A(g) A(h) A(k)) \\
& +C(A(f), A(g)) A(h) A(k)+C(A(f), A(h)) A(g) A(k)+\cdots \quad(6 \text { terms }) \\
& =\operatorname{Nor}(A(f) A(g) A(h) A(k) \\
& +C(A(f), A(g)) A(h) A(k)+C(A(f), A(h)) A(g) A(k)+\cdots)
\end{aligned}
$$

(6 ordered terms with 1 contraction)

$$
+C(A(f), A(g)) C(A(h), A(k))+C(A(f), A(h)) C(A(g), A(k))+\cdots
$$

(6 ordered terms with 2 contractions))

Applying Wick's theorem to $\mathcal{H}_{\text {red }}$ (putting the quartic and cubic terms in normal order, but not the quadratics) we get

$$
\begin{aligned}
& \mathcal{H}_{\text {red }}=N \mu_{0}(t) \\
& +N^{1 / 2} \int d x\left\{\tilde{h}(\phi(t, x)) b_{x}^{*}+\overline{\tilde{h}}(\phi(t, x)) b_{x}\right\} \\
& +\frac{1}{i} \frac{\partial}{\partial t}\left(e^{\mathcal{B}(k(t))}\right) e^{-\mathcal{B}(k(t)}+\int d x b_{x}^{*} \Delta b_{x} \\
& -\frac{1}{2} \int d x d y v_{N}(x-y)\left(\bar{\Lambda}(x, y) b_{x} b_{y}+\Lambda(x, y) b_{x}^{*} b_{y}^{*}+2 \bar{\Gamma}(x, y) b_{x}^{*} b_{y}\right) \\
& -\int d x\left(v_{N} * \operatorname{Tr} \Gamma\right) b_{x}^{*} b_{x} \\
& -\frac{1}{\sqrt{N}} \int d x_{1} d x_{2} \operatorname{Nor}\left\{v_{N}\left(x_{1}-x_{2}\right)\left(\bar{\phi}\left(x_{2}\right) b_{x_{1}}^{*} b_{x_{1}} b_{x_{2}}+\phi\left(x_{2}\right) b_{x_{1}}^{*} b_{x_{2}}^{*} b_{x_{1}}\right)\right\} \\
& -\frac{1}{2 N} \int d x_{1} d x_{2} \operatorname{Nor}\left\{v_{N}\left(x_{1}-x_{2}\right) b_{x_{1}}^{*} b_{x_{2}}^{*} b_{x_{1}} b_{x_{2}}\right\} .
\end{aligned}
$$

where $\tilde{h}$ is the modified Hartree operator (23). Let us remark that, in complete analogy to $(67 \mathrm{c})$ in [23], the quadratic terms (89a) $+(89 \mathrm{~b})+$ (89c) can be written concisely and explicitly as

$$
(\underline{89 \mathrm{a}})+(\underline{89 \mathrm{~b}})+(\underline{89 \mathrm{c}})=\mathcal{H}_{\tilde{G}}-\mathcal{I}\left(\begin{array}{cc}
\tilde{w}^{T} & \overline{\tilde{f}} \\
-\tilde{f} & -\tilde{w}
\end{array}\right)
$$


where

$$
\begin{aligned}
& \tilde{f}:=\left(\tilde{\mathbf{S}}(\operatorname{sh}(k))+\overline{\operatorname{ch}(k)} \circ\left(v_{N} \Lambda\right)\right) \circ \operatorname{ch}(k)-\left(\tilde{\mathbf{W}}(\overline{\operatorname{ch}(k)})-\operatorname{sh}(k) \circ \overline{\left(v_{N} \Lambda\right)}\right) \circ \operatorname{sh}(k) \\
& \tilde{w}:=\left(\tilde{\mathbf{W}}(\overline{\operatorname{ch}(k)})-\operatorname{sh}(k) \circ \overline{\left(v_{N} \Lambda\right)}\right) \circ \overline{\operatorname{ch}(k)}-\left(\tilde{\mathbf{S}}(\operatorname{sh}(k))+\overline{\operatorname{ch}(k)} \circ\left(v_{N} \Lambda\right)\right) \circ \overline{\operatorname{sh}(k)} \\
& \mathcal{H}_{\tilde{g}}=\int \tilde{g}(x, y) a_{x}^{*} a_{y} d x d y
\end{aligned}
$$

Also, $\mathcal{I}$ is the Lie algebra isomorphism used in our previous papers [21]-24] (see for instance formula (27) in [23]) is

$\mathcal{I}\left(\begin{array}{cc}\tilde{w}^{T} & \overline{\tilde{f}} \\ -\tilde{f} & -\tilde{w}\end{array}\right)=-\frac{1}{2} \int d x d y\left\{\tilde{w}(y, x) a_{x} a_{y}^{*}+\tilde{w}(x, y) a_{x}^{*} a_{y}-\tilde{f}(x, y) a_{x}^{*} a_{y}^{*}-\overline{\tilde{f}}(x, y) a_{x} a_{y}\right\}$

In this notation, $\tilde{X}_{2}$ is a multiple of $\tilde{f}$, thus $\tilde{f}=0$ if our equations are satisfied.

If we also put the quadratics in normal order, the above formula becomes

$$
\begin{aligned}
& \mathcal{H}_{\text {red }}=X_{0}(t)+ \\
& N^{1 / 2} \int d x\left\{\tilde{h}(\phi(t, x)) b_{x}^{*}+\overline{\tilde{h}}(\phi(t, x)) b_{x}\right\} \\
& +\mathcal{H}_{\tilde{g}}+\operatorname{Nor}\left(\mathcal{I}\left(\begin{array}{cc}
\tilde{w}^{T} & \overline{\tilde{f}} \\
-\tilde{f} & -\tilde{w}
\end{array}\right)\right. \\
& -\frac{1}{\sqrt{N}} \int d x_{1} d x_{2}\left\{v_{N}\left(x_{1}-x_{2}\right)\left(\bar{\phi}\left(x_{2}\right) b_{x_{1}}^{*} b_{x_{1}} b_{x_{2}}+\phi\left(x_{2}\right) b_{x_{1}}^{*} b_{x_{2}}^{*} b_{x_{1}}\right)\right\} \\
& \left.-\frac{1}{2 N} \int d x_{1} d x_{2}\left\{v_{N}\left(x_{1}-x_{2}\right) b_{x_{1}}^{*} b_{x_{2}}^{*} b_{x_{1}} b_{x_{2}}\right\}\right) .
\end{aligned}
$$

where $X_{0}$ is written down explicitly in Section 6 on [24]. In conclusion, if $\phi$ and $k$ satisfy our equation $X_{1}=0, X_{2}=0$, then $\tilde{h}(\phi(t, x))=0$, 
$\tilde{f}=0$ and

$$
\begin{aligned}
\mathcal{P} & :=H_{\text {red }}-\mathcal{H}-X_{0} \\
& =\mathcal{H}_{\tilde{g}}-\mathcal{H}_{1}+\operatorname{Nor}\left(\mathcal{I}\left(\begin{array}{cc}
\tilde{w}^{T} & 0 \\
0 & -\tilde{w}
\end{array}\right)\right) \\
+ & \operatorname{Nor}\left(-\frac{1}{2 \sqrt{N}} \int d x_{1} d x_{2}\left\{v_{N}\left(x_{1}-x_{2}\right)\left(\bar{\phi}\left(x_{2}\right) b_{x_{1}}^{*} b_{x_{1}} b_{x_{2}}+\phi\left(x_{2}\right) b_{x_{1}}^{*} b_{x_{2}}^{*} b_{x_{1}}\right)\right\}\right. \\
& \left.-\frac{1}{2 N} \int d x_{1} d x_{2}\left\{v_{N}\left(x_{1}-x_{2}\right) b_{x_{1}}^{*} b_{x_{2}}^{*} b_{x_{1}} b_{x_{2}}\right\}\right) \\
& +\frac{1}{2 N} \int d x_{1} d x_{2}\left\{v_{N}\left(x_{1}-x_{2}\right) a_{x_{1}}^{*} a_{x_{2}}^{*} a_{x_{1}} a_{x_{2}}\right\}
\end{aligned}
$$

Remark that the third and fourth term are $-\frac{1}{\sqrt{N}} \operatorname{Nor}\left(e^{\mathcal{B}}[\mathcal{A}, \mathcal{V}] e^{-\mathcal{B}}\right)$ and $-\frac{1}{N} \operatorname{Nor}\left(e^{\mathcal{B}} \mathcal{V} e^{-\mathcal{B}}\right)$ (see Section 5 of [23]).

\section{Estimates FOR THE ERROR TERM}

In this section we apply the estimates of Corollary (7.2) in order to estimate the error.

We proceed as in our previous papers [21]-[23], using the identity

$$
\left\|\psi_{\text {exact }}(t)-e^{i \int X_{0}(t) d t} \psi_{\text {appr }}(t)\right\|_{\mathcal{F}}=\left\|e^{-i \int X_{0}(t) d t} \psi_{\text {red }}-\Omega\right\|_{\mathcal{F}}
$$

and estimate the right hand side term using the equation

$$
\left(\frac{1}{i} \frac{\partial}{\partial t}-\mathcal{H}_{r e d}+X_{0}\right)\left(e^{-i \int X_{0}(t) d t} \psi_{r e d}-\Omega\right)=\left(0,0,0, X_{3}, X_{4}, 0, \cdots\right):=\tilde{X}
$$

Recall $X_{0}$ is the zeroth order term in $\mathcal{H}_{\text {red }}$.

Denote $E=e^{-i \int X_{0}(t) d t} \psi_{\text {red }}-\Omega$, so that

$$
\begin{aligned}
& \left(\frac{1}{i} \frac{\partial}{\partial t}-\mathcal{H}_{\text {red }}+X_{0}\right) E=\left(0,0,0, X_{3}, X_{4}, 0, \cdots\right):=\tilde{X} \\
& E(0, \cdot)=0
\end{aligned}
$$

In the proof that follows we will write

$$
\begin{aligned}
\mathbf{S}_{F} & :=\frac{1}{i} \frac{\partial}{\partial t}-\mathcal{H}_{\text {red }}+X_{0} \\
& :=\mathbf{S}_{D}-\mathcal{P}
\end{aligned}
$$

where

$$
\begin{aligned}
& \mathbf{S}_{D}=\frac{1}{i} \frac{\partial}{\partial t}-\mathcal{H} \\
& \mathcal{H}=\int a_{x}^{*} \Delta a_{x}-\frac{1}{2 N} \mathcal{V}
\end{aligned}
$$


Thus $\mathcal{H}$ is the original (unconjugated) Fock space Hamiltonian (8a)$(8 \mathrm{c})$, and $\mathcal{P}$ accounts for the rest of the terms:

$$
\mathcal{P}=H_{\text {red }}-\mathcal{H}-X_{0}
$$

Recall $\mathcal{H}$ acts on Fock space as

$$
\Delta-\frac{1}{N} \sum_{j<k} N^{3 \beta} v\left(N^{\beta}\left(x_{j}-x_{k}\right)\right)
$$

interpreted as 0 on the zeroth slot and $\Delta$ on the first one.

The terms $X_{0}, X_{3}$ and $X_{4}$ were computed in Section 5 of [23], see formulas (74c) and (72c). We only need $X_{3}$ and $X_{4}$ here, and they are (modulo symmetrization and normalization)

$$
\begin{aligned}
X_{3}\left(y_{1}, y_{2}, y_{3}\right)= & \frac{1}{\sqrt{N}} \int \overline{\operatorname{ch}}\left(y_{1}, x_{1}\right) \operatorname{ch}\left(x_{2}, y_{2}\right) v_{N}\left(x_{1}-x_{2}\right) \phi\left(x_{2}\right) \operatorname{sh}\left(y_{3}, x_{1}\right) d x_{1} d x_{2} \\
& =\frac{1}{\sqrt{N}}\left(v_{N}\left(y_{1}-y_{2}\right) \operatorname{sh}\left(y_{3}, y_{1}\right) \phi\left(y_{2}\right)+L O T\right) \\
X_{4}\left(y_{1}, y_{2}, y_{3}, y_{4}\right)= & \frac{1}{N} \int \overline{\operatorname{ch}}\left(y_{1}, x_{1}\right) \operatorname{ch}\left(x_{2}, y_{2}\right) v_{N}\left(x_{1}-x_{2}\right) \operatorname{sh}\left(y_{3}, x_{1}\right) \operatorname{sh}\left(x_{2}, y_{4}\right) d x_{1} d x_{2} \\
& =\frac{1}{N} v_{N}\left(y_{1}-y_{2}\right)\left(\operatorname{sh}\left(y_{3}, y_{1}\right) \operatorname{sh}\left(y_{2}, y_{4}\right)+L O T\right)
\end{aligned}
$$

where the lower order terms LOT come from the $p$ component of $\operatorname{ch}(k)=\delta(x-y)+p$.

We will use the following Strichartz norms $S$ and dual Strichartz norms $S^{\prime}$ for the equation $\mathbf{S} u=f$ where $u=u\left(t, x_{1}, \cdots x_{n}\right), x_{i} \in \mathbb{R}^{3}$, $t \in\left[0, T_{0}\right]$.

Definition 9.1. Define the following norms for $0 \leq t \leq T_{0}$ :

$$
\|u\|_{S}=\max \left\{\|u\|_{L^{\infty}(d t) L^{2}\left(d x_{1} \cdots d x_{n}\right)},\|u\|_{L^{2}(d t) L^{6}\left(d\left(x_{1}-x_{2}\right)\right) L^{2}\left(d\left(x_{1}+x_{2}\right) \cdots d x_{n}\right)},\right.
$$

and all other permutaions

and

$$
\|u\|_{S^{\prime}}=\min \left\{\|u\|_{L^{1}(d t) L^{2}\left(d x_{1} \cdots d x_{n}\right)},\|u\|_{L^{2}(d t) L^{6 / 5}\left(d\left(x_{1}-x_{2}\right)\right) L^{2}\left(d\left(x_{1}+x_{2}\right) \cdots d x_{n}\right)},\right.
$$

and all other permutaions $\}$

Also, if $X$ is an element of Fock space with finitely many non-zero components $X_{0}, \cdots, X_{k}$, we denote

$$
\|X\|_{S}=\max \left\{\left|X_{0}\right|,\left\|X_{1}\right\|_{S}, \cdots,\left\|X_{k}\right\|_{S}\right\},
$$

and similarly

$$
\|X\|_{S^{\prime}}=\max \left\{\left|X_{0}\right|,\left\|X_{1}\right\|_{S^{\prime}}, \cdots,\left\|X_{k}\right\|_{S^{\prime}}\right\}
$$


As it is well known, using the $T-T^{*}$ argument and the Christ-Kiselev lemma, as well as estimates for the homogeneous equation such as (57) if $\mathbf{S} u=f, u(t=0)=0$, then $\|u\|_{S} \lesssim\|f\|_{S^{\prime}}$.

Furthermore, if $\beta<1$,

$$
\begin{aligned}
& \frac{1}{N}\left\|v_{N}\left(x_{1}-x_{2}\right) u\right\|_{S^{\prime}} \leq \frac{1}{N}\left\|v_{N}\left(x_{1}-x_{2}\right) u\right\|_{L^{2}(d t) L^{6 / 5}\left(d\left(x_{1}-x_{2}\right)\right) L^{2}\left(d\left(x_{1}+x_{2}\right) \cdots d x_{n}\right)} \\
& \leq \frac{1}{N}\left\|v_{N}\right\|_{L^{3 / 2}}\|u\|_{L^{2}(d t) L^{6}\left(d\left(x_{1}-x_{2}\right)\right) L^{2}\left(d\left(x_{1}+x_{2}\right) \cdots d x_{n}\right)}, \\
& \leq C N^{- \text {power }}\|u\|_{S}
\end{aligned}
$$

so we can treat the potential as a perturbation and conclude the following:

Lemma 9.2. Let $f$ be a Fock vector with zero entries past the $k$ th slot ( $k=21$ in the application that follows). Assume

$$
\begin{aligned}
& \mathbf{S}_{D} u=f \\
& u(t=0)=0
\end{aligned}
$$

then

$$
\|u\|_{S} \lesssim\|f\|_{S^{\prime}}
$$

and notice that $\sup _{t}\|u\|_{\mathcal{F}} \lesssim\|u\|_{S}$.

The main result of this section, which completes the proof of Theorem (2.5), is

Theorem 9.3. Let $\beta<2 / 3$, and let $E$ satisfy (911). Assume $\Lambda$, $\phi$ and $\Gamma$ satisfy the estimates of Theorem (6.1) as well as Corollary (7.2). Then

$$
\|E\|_{\mathcal{F}} \lesssim N^{-\frac{1}{6}}
$$

for $0 \leq t \leq T_{0}$.

Proof. Recall the splitting

$$
\begin{aligned}
\mathbf{S}_{F} & :=\frac{1}{i} \frac{\partial}{\partial t}-\mathcal{H}_{\text {red }}+X_{0} \\
& :=\mathbf{S}_{D}-\mathcal{P}
\end{aligned}
$$

where $\mathbf{S}_{D}$ is a diagonal Schrödinger operator. Also, $\mathcal{P}$ was computed at the end of Section (8) .

We observe $\left\|X_{4}\right\|_{L^{2}}=c N^{\frac{3 \beta}{2}-1}<<1$, but $\left\|X_{3}\right\|_{L^{2}}=c N^{\frac{3 \beta-1}{2}}>>1$ so we cannot use energy estimates and are forced to use the Strichartz 
estimates of Lemma (9.2). We proceed to solve

$$
\begin{aligned}
& \left(S_{D}-\mathcal{P}\right) E=\tilde{X}=\left(0,0,0, X_{3}, X_{4}, \cdots\right) \\
& E(0)=0
\end{aligned}
$$

by iteration. We will iterate 4 times, and by the end the vector on the right hand side will have at most $5+4 \times 4$ non-zero entries. $(\mathcal{P}$ is a fourth order operator in $a$ and $a^{*}$ ).

Since $\left\|\frac{1}{\sqrt{N}} v_{N}\right\|_{L^{6 / 5}}=c N^{\frac{\beta-1}{2}} \leq c N^{-\frac{1}{6}}$, we have $\|X\|_{S^{\prime}} \lesssim N^{-\frac{1}{6}}$ and therefore the first iterate defined by

$$
\begin{aligned}
& S_{D} E_{1}=\tilde{X}=\left(0,0,0, X_{3}, X_{4}, \cdots\right) \\
& E_{1}(0)=0
\end{aligned}
$$

satisfies $\left\|E_{1}\right\|_{S} \lesssim N^{-\frac{1}{6}}$. If $\mathcal{P}$ were bounded on the first few slots of Fock space we would be done. Unfortunately, this is not the case, the norm of $\mathcal{P}$ on the first few slots of Fock space is $\leq C N^{\frac{3 \beta-1}{2}} \leq C N^{\frac{1}{2}}$.

In Section (10) below we prove that $\mathcal{P}=\mathcal{P}_{1}+\mathcal{P}_{2}$ where $\mathcal{P}_{2}$ is bounded on (the first 21 slots of) Fock space, while $\left\|\mathcal{P}_{1} E\right\|_{\mathcal{F}} \lesssim N^{\frac{1}{2}}\|E\|_{\mathcal{F}}$ but also $\left\|\mathcal{P}_{1} E\right\|_{S} \lesssim N^{-\frac{1}{6}}\|E\|_{S^{\prime}}$. The reasons behind these bounds are $\left\|\frac{1}{\sqrt{N}} v_{N}\right\|_{L^{6 / 5}}=c N^{\frac{\beta-1}{2}} \leq c N^{-\frac{1}{6}},\left\|\frac{1}{\sqrt{N}} v_{N}\right\|_{L^{2}}=c N^{\frac{3 \beta}{2}-1} \leq c N^{\frac{1}{2}}$. Consider the iteration

$$
\begin{aligned}
& \left(S_{D}-\mathcal{P}\right)\left(E_{1}+\mathbf{S}_{D}^{-1} \mathcal{P}_{1} E_{1}+\left(\mathbf{S}_{D}^{-1} \mathcal{P}_{1}\right)^{2} E_{1}+\left(\mathbf{S}_{D}^{-1} \mathcal{P}_{1}\right)^{3} E_{1}\right) \\
& =\tilde{X}-\mathcal{P}_{2}\left(E_{1}+\mathbf{S}_{D}^{-1} \mathcal{P}_{1} E_{1}+\left(\mathbf{S}_{D}^{-1} \mathcal{P}_{1}\right)^{2} E_{1}\right)-\mathcal{P}\left(\mathbf{S}_{D}^{-1} \mathcal{P}_{1}\right)^{3} E_{1}
\end{aligned}
$$

Assuming the estimates of Propositions (10.1), (10.3), (10.4), we have the following fixed time estimates for $0 \leq t \leq T_{0}$ :

$$
\begin{aligned}
& \left\|\mathcal{P}_{2}\left(E_{1}+\mathbf{S}_{D}^{-1} \mathcal{P}_{1} E_{1}+\left(\mathbf{S}_{D}^{-1} \mathcal{P}_{1}\right)^{2} E_{1}\right)\right\|_{\mathcal{F}} \\
& \lesssim\left\|\left(E_{1}+\mathbf{S}_{D}^{-1} \mathcal{P}_{1} E_{1}+\left(\mathbf{S}_{D}^{-1} \mathcal{P}_{1}\right)^{2} E_{1}\right)\right\|_{\mathcal{F}} \\
& \lesssim N^{-\frac{1}{6}}
\end{aligned}
$$

and

$$
\left\|\mathcal{P}\left(\mathbf{S}_{D}^{-1} \mathcal{P}_{1}\right)^{3} E_{1}\right\|_{\mathcal{F}} \lesssim N^{\frac{1}{2}} N^{-\frac{4}{6}} \lesssim N^{-\frac{1}{6}}
$$

Now we can use energy estimates to compare the above fourth iterate with the exact solution to (91) as in our previous papers [21]-[23], and conclude the proof of the theorem.

All we have to do now is estimate the norm $\mathcal{P}$, restricted to the first 21 slots of Fock space. In order to do that, we need some precise information on the terms in $\mathcal{P}$, which was developed in Section (8) . 


\section{Estimates FOR THE NORM OF $\mathcal{P}$ RESTRICTED TO THE FIRST 21 SLOTS OF FOCK SPACE}

In this section we will use repeatedly the fact that, if $\beta \leq 2 / 3$, $\frac{1}{N}\left\|v_{N}\right\|_{L^{2}} \lesssim 1, \frac{1}{\sqrt{N}}\left\|v_{N}\right\|_{L^{2}} \lesssim N^{\frac{1}{2}}, \frac{1}{\sqrt{N}}\left\|v_{N}\right\|_{L^{\frac{6}{5}}} \lesssim N^{-\frac{1}{6}}$. Recall formula (90) defining $\mathcal{P}$..

We start by defining the term $\mathcal{P}_{1}$ and proving its properties.

Proposition 10.1. Let $\mathcal{P}_{1}$ is a linear combination of the following terms coming from

$$
\begin{gathered}
\frac{1}{\sqrt{N}} \operatorname{Nor}\left(e^{\mathcal{B}}[\mathcal{A}, \mathcal{V}] e^{-\mathcal{B}}\right) \\
T_{1}=\frac{1}{\sqrt{N}} \int d x_{1} d x_{2} v_{N}\left(x_{1}-x_{2}\right) \bar{\phi}\left(x_{2}\right) a\left(\overline{\operatorname{sh}(k)}\left(x_{1}, \cdot\right)\right) a\left(\overline{\operatorname{ch}(k)}\left(x_{1}, \cdot\right)\right) a\left(\overline{\operatorname{ch}(k)}\left(x_{2}, \cdot\right)\right) \\
T_{2}=\frac{1}{\sqrt{N}} \int d x_{1} d x_{2} v_{N}\left(x_{1}-x_{2}\right) \phi\left(x_{2}\right) a^{*}\left(\operatorname{sh}(k)\left(x_{1}, \cdot\right)\right) a^{*}\left(\operatorname{ch}(k)\left(x_{1}, \cdot\right)\right) a^{*}\left(\operatorname{ch}(k)\left(x_{2}, \cdot\right)\right) \\
T_{3}=\frac{1}{\sqrt{N}} \int d x_{1} d x_{2} v_{N}\left(x_{1}-x_{2}\right) \bar{\phi}\left(x_{2}\right) a^{*}\left(\operatorname{ch}(k)\left(x_{1}, \cdot\right)\right) a\left(\overline{\operatorname{ch}(k)}\left(x_{1}, \cdot\right)\right) a\left(\overline{\operatorname{ch}(k)}\left(x_{2}, \cdot\right)\right) \\
T_{4}=\frac{1}{\sqrt{N}} \int d x_{1} d x_{2} v_{N}\left(x_{1}-x_{2}\right) \phi\left(x_{2}\right) a^{*}\left(\operatorname{ch}(k)\left(x_{1}, \cdot\right)\right) a^{*}\left(\operatorname{ch}(k)\left(x_{2}, \cdot\right)\right) a\left(\overline{(\operatorname{ch}(k)}\left(x_{1}, \cdot\right)\right)
\end{gathered}
$$

If $X$ is a Fock space vector which has non-zero entries only in the first $k(k=21)$ slots and $T$ is one of the above $T_{i}$, then

$$
\begin{aligned}
\|T X\|_{\mathcal{F}} & \lesssim N^{\frac{1}{2}}\|X\|_{\mathcal{F}} \\
\|T X\|_{S^{\prime}} & \lesssim N^{-\frac{1}{6}}\|X\|_{S}
\end{aligned}
$$

Remark 10.2. It is easy to see the meaning of these terms. $T_{1}^{*}=T_{2}$, $T_{2} \Omega=X_{3}$ while $T_{3}$ and $T_{4}$ with $\operatorname{ch}(k)$ replaced by $\delta(x-y)$ correspond to the unconjugated $\frac{1}{\sqrt{N}}[\mathcal{A}, \mathcal{V}]$.

Proof. In treating the above terms recall $\operatorname{ch}(k)(t, x, y)=\delta(x-y)+$ $p(t, x, y)$. The worst terms are always obtained from the $\delta$ term (because composition with $p$ is bounded on $L^{2}$ ), so we will only discuss these. Also, recall $\phi$ is known to be bounded, and $L^{2}$. Replacing $\operatorname{ch}(k)(t, x, y)$ by $\delta(x-y), T_{1}$ gets replaced by

$$
\frac{1}{\sqrt{N}} \int d x_{1} d x_{2} v_{N}\left(x_{1}-x_{2}\right) \bar{\phi}\left(x_{2}\right) a\left(\overline{\operatorname{sh}(k)}\left(x_{1}, \cdot\right)\right) a_{x_{1}} a_{x_{2}}
$$


This acts on a Fock space vector of the form $\left(0, \cdots, F\left(x_{1}, \cdots x_{n}, 0, \cdots\right)\right.$ as

$$
\left.\int \frac{1}{\sqrt{N}} v_{N}\left(x_{1}-x_{2}\right) \bar{\phi}\left(x_{2}\right) \overline{\operatorname{sh}(k)}\left(x_{1}, z\right)\right) F\left(x_{1}, x_{2}, z, \cdots\right) d x_{1} d x_{2} d z
$$

Now we use

$$
\begin{aligned}
& \left.\| \frac{1}{\sqrt{N}} v_{N}\left(x_{1}-x_{2}\right) \bar{\phi}\left(x_{2}\right) \overline{\operatorname{sh}(k)}\left(x_{1}, z\right)\right) \|_{L^{2}\left(d x_{1} d x_{2} d z\right)} \\
& \leq \sup _{x_{1}}\left\|\overline{\operatorname{sh}(k)}\left(x_{1}, z\right)\right\|_{L^{2}(d z)}\|\| \frac{1}{\sqrt{N}} v_{N}\left\|_{L^{2}}\right\| \phi \|_{L^{2}} \lesssim N^{\frac{1}{2}}
\end{aligned}
$$

which implies (93), and also

$$
\begin{aligned}
& \left.\| \frac{1}{\sqrt{N}} v_{N}\left(x_{1}-x_{2}\right) \bar{\phi}\left(x_{2}\right) \overline{\operatorname{sh}(k)}\left(x_{1}, z\right)\right) \|_{L^{6 / 5}\left(d\left(x_{1}-x_{2}\right)\right) L^{2}\left(\left(d x_{1}+x_{2}\right) d z\right)} \\
& \leq \sup _{x_{1}}\left\|\overline{\operatorname{sh}(k)}\left(x_{1}, z\right)\right\|_{L^{2}(d z)}\|\| \frac{1}{\sqrt{N}} v_{N}\left\|_{L^{6 / 5}}\right\| \phi \|_{L^{2}} \lesssim N^{-\frac{1}{6}}
\end{aligned}
$$

which implies the fixed time estimate

$$
\left\|T_{1}(F)(t)\right\|_{L^{2}} \lesssim N^{-\frac{1}{6}}\|F\|_{L^{6}\left(d\left(x_{1}-x_{2}\right)\right) L^{2}\left(d\left(x_{1}+x_{2}\right) d x_{3} \cdots\right)}
$$

Now take $L^{1}$ in time, and dominate that by $L^{2}$ in time on the right hand side, since $t \in[0,1]$. This proves

$$
\left\|T_{1}(F)(t)\right\|_{S^{\prime}} \lesssim N^{-\frac{1}{6}}\|F\|_{S}
$$

The estimate for $T_{2}$ is the dual of this argument. The term $T_{3}$ is (after replacing $\operatorname{ch}(k)$ by $\delta)$

$$
\frac{1}{\sqrt{N}} \int d x_{1} d x_{2} v_{N}\left(x_{1}-x_{2}\right) \bar{\phi}\left(x_{2}\right) a_{x_{1}}^{*} a_{x_{1}} a_{x_{2}}
$$

This acts on $F$ by

$$
\frac{1}{\sqrt{N}} \int v_{N}\left(x_{1}-x_{2}\right) \bar{\phi}\left(x_{2}\right) F\left(x_{1}, x_{2}, \cdots\right) d x_{2}
$$

The variables $x_{j}, j \geq 3$ are passive, so, without loss of generality, we take $F=F\left(x_{1}, x_{2}\right)$ The $L^{2}$ bound is immediate, and for the $S, S^{\prime}$ bound write $F\left(x_{1}, x_{2}\right)=G\left(x_{1}-x_{2}, x_{1}+x_{2}\right)$ and

$$
\begin{aligned}
& \frac{1}{\sqrt{N}}\left\|\int v_{N}\left(x_{1}-x_{2}\right) \bar{\phi}\left(x_{2}\right) G\left(x_{1}-x_{2}, x_{1}+x_{2}\right) d x_{2}\right\|_{L^{2}\left(d x_{1}\right)} \\
& =\frac{1}{\sqrt{N}}\left\|\int v_{N}\left(x_{2}\right) \bar{\phi}\left(x_{1}-x_{2}\right) G\left(x_{2}, 2 x_{1}-x_{2}\right) d x_{2}\right\|_{L^{2}\left(d x_{1}\right)} \\
& \leq \frac{1}{\sqrt{N}}\|\phi\|_{L^{\infty}}\left\|v_{N}\right\|_{L^{6 / 5}}\|G\|_{L^{6} L^{2}}=c N^{-\frac{1}{6}}\|\phi\|_{L^{\infty}}\|F\|_{L^{6}(d(x-y)) L^{2}(d(x+y))}
\end{aligned}
$$


The bounds for $T_{4}$ are easy, and left to the reader.

Proposition 10.3. The terms of $\mathcal{P}$ other than $T_{1}, \cdots T_{4}$ coming from

$$
\frac{1}{\sqrt{N}} \operatorname{Nor}\left(e^{\mathcal{B}}[\mathcal{A}, \mathcal{V}] e^{-\mathcal{B}}\right)
$$

have bounded operator norm on the first 21 slots of Fock space.

Proof. To prove the proposition, we have to estimate the terms in

$$
\frac{1}{\sqrt{N}} \operatorname{Nor}\left(\int d x_{1} d x_{2}\left\{v_{N}\left(x_{1}-x_{2}\right)\left(\bar{\phi}\left(x_{2}\right) b_{x_{1}}^{*} b_{x_{1}} b_{x_{2}}+\phi\left(x_{2}\right) b_{x_{1}}^{*} b_{x_{2}}^{*} b_{x_{1}}\right)\right\}\right)
$$

The two terms are dual to each other, so will just estimate the first one. In principle, there are $2^{3}$ terms to estimate, following the pattern

$$
\begin{array}{r}
a_{1} a_{1} a_{2} \\
a_{1}^{*} a_{1} a_{2} \\
a_{1} a_{1}^{*} a_{2} \\
\ldots \\
a_{1}^{*} a_{1} a_{2}^{*} \\
\ldots
\end{array}
$$

The two terms, (95a) and (95b) are $T_{1}$ and $T_{3}$, which have been discussed in the previous lemma. During the proof, we will comment on where some of the contraction terms go, but point out we do not need to estimate them.

The term (95c) stands for

$\frac{1}{\sqrt{N}} \int d x_{1} d x_{2} v_{N}\left(x_{1}-x_{2}\right) \bar{\phi}\left(x_{2}\right) a\left(\overline{\operatorname{sh}(k)}\left(x_{1}, \cdot\right)\right) a^{*}\left(\operatorname{sh}(k)\left(x_{1}, \cdot\right)\right) a\left(\overline{\operatorname{ch}(k)}\left(x_{2}, \cdot\right)\right)$

Here the contraction $\left[a\left(\overline{\operatorname{sh}(k)}\left(x_{1}, \cdot\right)\right), a^{*}\left(\operatorname{sh}(k)\left(x_{1}, \cdot\right)\right)\right]=(\overline{\operatorname{sh}(k)} \circ \operatorname{sh}(k))\left(x_{1}, x_{1}\right)$ pairs up with $|\phi|^{2}$ to form $\operatorname{Tr} \Gamma$ in the formula for $\tilde{h}$, and we do not have to estimate it. The ordered term

$\frac{1}{\sqrt{N}} \int d x_{1} d x_{2} v_{N}\left(x_{1}-x_{2}\right) \bar{\phi}\left(x_{2}\right) a^{*}\left(\operatorname{sh}(k)\left(x_{1}, \cdot\right) a\left(\overline{\operatorname{sh}(k)}\left(x_{1}, \cdot\right)\right)\right) a\left(\overline{\operatorname{ch}(k)}\left(x_{2}, \cdot\right)\right)$ acts on $F$ as

$\left.\frac{1}{\sqrt{N}} \int v_{N}\left(x_{1}-x_{2}\right) \bar{\phi}\left(x_{2}\right) \operatorname{sh}(k)\left(x_{1}, x_{3}\right) \overline{\operatorname{sh}(k)}\left(x_{1}, z\right)\right) F\left(x_{2}, z, \cdots\right) d x_{1} d x_{2} d z$ and has operator norm $\lesssim \frac{1}{\sqrt{N}}$.

The term (95d) stands for

$$
\frac{1}{\sqrt{N}} \int d x_{1} d x_{2} \bar{\phi}\left(x_{2}\right) a^{*}\left(\operatorname{ch}(k)\left(x_{1}, \cdot\right)\right) a\left(\overline{\operatorname{ch}(k)}\left(x_{1}, \cdot\right)\right) a^{*}\left(\operatorname{sh}(k)\left(x_{2}, \cdot\right)\right)
$$


Here the estimate is not true for the term involving the contraction $\left[a\left(\overline{\operatorname{ch}(k)}\left(x_{1}, \cdot\right)\right), a^{*}\left(\operatorname{sh}(k)\left(x_{2}, \cdot\right)\right)\right]=\frac{1}{2} \operatorname{sh}(2 k)\left(x_{1}, x_{2}\right)$. This term gets paired up with $\phi\left(x_{1}\right) \phi\left(x_{2}\right)$ to form $\Lambda$, and becomes part of $\tilde{h}(\phi)$. The remaining ordered term has norm $\lesssim \frac{1}{\sqrt{N}}$.

All remaining terms have operator norms $\lesssim \frac{1}{\sqrt{N}}$, as can be easily checked.

Proposition 10.4. The terms $\mathcal{P}$ coming from

$$
\frac{1}{2 N} \operatorname{Nor}\left(e^{\mathcal{B}} \mathcal{V} e^{-\mathcal{B}}\right)-\frac{1}{2 N} \mathcal{V}
$$

have bounded operator norm on the first 21 slots of Fock space.

Proof. We have to estimate the $2^{4}-1$ terms in

$\frac{1}{2 N} \operatorname{Nor}\left(\int v_{N}\left(x_{1}-x_{2}\right) b_{x_{1}}^{*} b_{x_{2}}^{*} b_{x_{1}} b_{x_{2}} d x_{1} d x_{2}-\frac{1}{2 N} \int v_{N}\left(x_{1}-x_{2}\right) a_{x_{1}}^{*} a_{x_{2}}^{*} a_{x_{1}} a_{x_{2}} d x_{1} d x_{2}\right)$,

During the proof, we will comment on where some of the contraction terms go, but point out we do not need to estimate them. Recall the formula (87).

The operators $b_{x}-a_{x}$ and $b_{x}^{*}-a_{x}^{*}$ are linear combinations of $a(f(x, \cdot)$, $a^{*}(f(x, \cdot)$ where $f$ is one of $\operatorname{sh}(k), p$, or their complex conjugates, and satisfies the estimates of Lemma (7.2).

We look at all possible terms in $b_{x_{1}}^{*} b_{x_{2}}^{*} b_{x_{1}} b_{x_{2}}$. Schematically,

$$
\begin{array}{r}
a_{1} a_{2} a_{1} a_{2} \\
a_{1}^{*} a_{2} a_{1} a_{2} \\
a_{1} a_{2}^{*} a_{1} a_{2} \\
a_{1}^{*} a_{2}^{*} a_{1} a_{2} \\
\cdots \\
a_{1}^{*} a_{2}^{*} a_{1}^{*} a_{2} \\
a_{1}^{*} a_{2}^{*} a_{1} a_{2}^{*} \\
\cdots \\
a_{1}^{*} a_{2}^{*} a_{1}^{*} a_{2}^{*}
\end{array}
$$

and estimate some typical ones.

The term (96a) means

$$
\frac{1}{2 N} \int v_{N}\left(x_{1}-x_{2}\right) a\left(\overline { \operatorname { s h } ( k ) } ( x _ { 1 } , \cdot ) a \left(\overline { \operatorname { s h } ( k ) } ( x _ { 2 } , \cdot ) a \left(\overline { \operatorname { c h } ( k ) } ( x _ { 1 } , \cdot ) a \left(\overline{\operatorname{ch}(k)}\left(x_{2}, \cdot\right) d x_{1} d x_{2}\right.\right.\right.\right.
$$


and this is dual to $96 \mathrm{~g}$ )

$\frac{1}{2 N} \int v_{N}\left(x_{1}-x_{2}\right) a^{*}\left(\operatorname{ch}(k)\left(x_{1}, \cdot\right) a^{*}\left(\operatorname{ch}(k)\left(x_{2}, \cdot\right) a^{*}\left(\operatorname{sh}(k)\left(x_{1}, \cdot\right) a^{*}\left(\operatorname{sh}(k)\left(x_{2}, \cdot\right) d x_{1} d x_{2}\right.\right.\right.\right.$

The first one acts by integration against, and the second one acts as a (normalized, symmetrized) tensor product with

$X_{4}\left(y_{1}, y_{2}, y_{3}, y_{4}\right)=\frac{1}{N} \int \overline{\operatorname{ch}}\left(y_{1}, x_{1}\right) \operatorname{ch}\left(x_{2}, y_{2}\right) v_{N}\left(x_{1}-x_{2}\right) \operatorname{sh}\left(y_{3}, x_{1}\right) \operatorname{sh}\left(x_{2}, y_{4}\right) d x_{1} d x_{2}$

Treating $\operatorname{ch}(k)\left(x_{1}, x_{2}\right)=\delta\left(x_{1}-x_{2}\right)+\mathrm{p}(k)$ we will only include $\delta$ in our calculation since this is always the worst case. With this simplification, the norm of the above operators is dominated by

$$
\begin{array}{r}
\left\|\frac{1}{2 N} v_{N}\left(y_{1}-y_{2}\right) \operatorname{sh}(k)\left(y_{3}, y_{1}\right) \operatorname{sh}(k)\left(y_{2}, y_{4}\right)\right\|_{L^{2}\left(d y_{1} d y_{2} d y_{3} d y_{4}\right)} \\
\leq\left\|\frac{1}{2 N} v_{N}\right\|_{L^{2}} \sup _{x}\|\operatorname{sh}(k)(x, y)\|_{L^{2}(d y)}\|\operatorname{sh}(k)(x, y)\|_{L^{2}(d x d y)} \lesssim 1
\end{array}
$$

Next we consider (96b) and the dual (96e). It suffices to treat just one, say (96e). This term stands for

$$
\frac{1}{2 N} \int v_{N}\left(x_{1}-x_{2}\right) a^{*}\left(\operatorname { c h } ( k ) ( x _ { 1 } , \cdot ) a ^ { * } \left(\operatorname { c h } ( k ) ( x _ { 2 } , \cdot ) a ^ { * } \left(\operatorname { s h } ( k ) ( x _ { 1 } , \cdot ) a \left(\overline{\operatorname{ch}(k)}\left(x_{2}, \cdot\right) d x_{1} d x_{2}\right.\right.\right.\right.
$$

For simplicity, replacing $\operatorname{ch}(k)\left(x_{1}, x_{2}\right)$ by $\delta\left(x_{1}-x_{2}\right)$, the above term acts on $F(\cdots)$ (actually, the vector $(0, \cdots, F, 0, \cdots)$ producing

$$
G\left(x_{1}, x_{2}, x_{3}, \cdots\right)=c \frac{1}{N} v_{N}\left(x_{1}-x_{2}\right) \operatorname{sh}(k)\left(x_{1}, x_{3}\right) F\left(x_{2}, \cdots\right)
$$

which is easily seen to have $L^{2}$ norm $\lesssim\|F\|_{L^{2}}$. (first do $L^{2}\left(d x_{3}\right)$, then $L^{2}\left(d x_{1}\right)$, leaving $L^{2}\left(d x_{2} d(\cdots)\right)$ last). Next we consider (96c) and the dual (96f), written explicitly as

$$
\begin{aligned}
& \frac{1}{2 N} \int v_{N}\left(x_{1}-x_{2}\right) a\left(\overline{\operatorname{sh}(k)}\left(x_{1}, \cdot\right)\right) a^{*}\left(\operatorname{ch}(k)\left(x_{2}, \cdot\right)\right) a\left(\overline{\operatorname{ch}(k)}\left(x_{1}, \cdot\right)\right) a\left(\overline{\operatorname{ch}(k)}\left(x_{2}, \cdot\right)\right) d x_{1} d x_{2} \\
& \frac{1}{2 N} \int v_{N}\left(x_{1}-x_{2}\right) a^{*}\left(\operatorname{ch}(k)\left(x_{1}, \cdot\right) a^{*}\left(\operatorname{ch}(k)\left(x_{2}, \cdot\right)\right) a\left(\overline{\operatorname{ch}(k)}\left(x_{1}, \cdot\right)\right) a^{*}\left(\operatorname{sh}(k)\left(x_{2}, \cdot\right)\right) d x_{1} d x_{2}\right.
\end{aligned}
$$

The estimate would not be true for these terms as they stand, but becomes true after putting them in normal order. For the first one, the contraction

$$
\frac{1}{2 N}\left[a\left(\overline{\operatorname{sh}(k)}\left(x_{1}, \cdot\right)\right), a^{*}\left(\operatorname{ch}(k)\left(x_{2}, \cdot\right)\right)\right]=\frac{1}{4 N} \overline{\operatorname{sh}(2 k)}\left(x_{1}, x_{2}\right)
$$


gets paired with $\bar{\phi}\left(x_{1}\right) \phi\left(x_{2}\right)$ from the quadratic term (88d) to become $\bar{\Lambda}\left(x_{1}, x_{2}\right)$. We are left with estimating

$\frac{1}{2 N} \int v_{N}\left(x_{1}-x_{2}\right) a^{*}\left(\operatorname{ch}(k)\left(x_{2}, \cdot\right)\right) a\left(\overline{\operatorname{sh}(k)}\left(x_{1}, \cdot\right)\right) a\left(\overline{\operatorname{ch}(k)}\left(x_{1}, \cdot\right)\right) a\left(\overline{\operatorname{ch}(k)}\left(x_{2}, \cdot\right)\right) d x_{1} d x_{2}$

With the usual simplification $\operatorname{ch}(k)=\delta+\cdots$ this acts on $F$ as

$$
\left.\frac{1}{2 N} \int v_{N}\left(x_{1}-x_{2}\right) \overline{\operatorname{sh}(k)}\left(x_{1}, z\right)\right) F\left(x_{1}, x_{2}, z \cdots\right) d x_{1} d z
$$

which is easily seen to have norm $\lesssim\|F\|_{L^{2}}$.

For (96d) we estimate the difference

$$
\begin{aligned}
& \frac{1}{2 N} \int v_{N}\left(x_{1}-x_{2}\right) a^{*}\left(\operatorname{ch}(k)\left(x_{1}, \cdot\right)\right) a^{*}\left(\operatorname{ch}(k)\left(x_{2}, \cdot\right)\right) a\left(\overline{\operatorname{ch}(k)}\left(x_{1}, \cdot\right)\right) a\left(\overline{\operatorname{ch}(k)}\left(x_{2}, \cdot\right)\right) d x_{1} d x_{2} \\
- & \frac{1}{2 N} \int v_{N}\left(x_{1}-x_{2}\right) a_{x_{1}}^{*} a_{x_{2}}^{*} a_{x_{1}} a_{x_{2}} d x_{1} d x_{2},
\end{aligned}
$$

For instance,

$\frac{1}{2 N} \int v_{N}\left(x_{1}-x_{2}\right) a^{*}\left(p\left(x_{1}, \cdot\right)\right) a^{*}\left(\operatorname{ch}(k)\left(x_{2}, \cdot\right)\right) a\left(\overline{\operatorname{ch}(k)}\left(x_{1}, \cdot\right)\right) a\left(\overline{\operatorname{ch}(k)}\left(x_{2}, \cdot\right)\right) d x_{1} d x_{2}$

replacing the $\operatorname{ch}(k)$ with $\delta$, acts by

$$
\frac{1}{2 N} \int v_{N}\left(x_{1}-x_{2}\right) p\left(x_{1}, z\right) F\left(x_{1}, x_{2}\right) d x_{1}
$$

which has $L^{2}\left(d x_{2} d z\right)$ norm $\lesssim\left\|\frac{1}{2 N} v_{N}\right\|_{L^{2}}\|p\|_{L^{\infty} L^{2}}\|F\|_{L^{2}} \lesssim\|F\|_{L^{2}}$.

All other terms are similar.

Lemma 10.5. Terms of $\mathcal{P}$ coming from

$$
\mathcal{H}_{\tilde{g}}-\mathcal{H}_{1}+\operatorname{Nor}\left(\mathcal{I}\left(\begin{array}{cc}
\tilde{w}^{T} & 0 \\
0 & -\tilde{w}
\end{array}\right)\right)
$$

are bounded from the first 21 slots of Fock space to Fock space uniformly in $N$.

Proof. Since $\Gamma$ is known to be bounded uniformly in $N$, this is clear for $\mathcal{H}_{\tilde{g}}-\mathcal{H}_{1}$ which equals

$\int\left(v_{N} *(\operatorname{Tr} \Gamma)(t, x) \delta(x-y)+v_{N}(x-y) \Gamma(t, x, y)\right) a_{x}^{*} a_{y} d x d y$. Also, recall

$$
\tilde{w}:=\left(\tilde{\mathbf{W}}(\overline{\operatorname{ch}(k)})-\operatorname{sh}(k) \circ \overline{\left(v_{N} \Lambda\right)}\right) \circ \overline{\operatorname{ch}(k)}-\left(\tilde{\mathbf{S}}(\operatorname{sh}(k))+\overline{\operatorname{ch}(k)} \circ\left(v_{N} \Lambda\right)\right) \circ \overline{\operatorname{sh}(k)}
$$


The equation $\tilde{f}=0$ implies the identity (see section 5 of [23] for a similar calculation) we get

$$
\begin{aligned}
& \tilde{w}(y, x)=\tilde{\mathbf{W}}(\overline{\operatorname{ch}(k)}) \circ(\overline{\operatorname{ch}(k)})^{-1}-\operatorname{sh}(k) \circ \overline{\left(v_{N} \Lambda\right)} \circ(\overline{\operatorname{ch}(k)})^{-1} \\
& =-\frac{1}{2}\left((\overline{\operatorname{ch}(k)})^{-1} \circ\left(v_{N} \Lambda\right) \circ \overline{\operatorname{sh}(k)}-\operatorname{sh}(k) \circ \overline{\left(v_{N} \Lambda\right)} \circ(\overline{\operatorname{ch}(k)})^{-1}\right) \\
& -\frac{1}{2}\left[\tilde{\mathbf{W}}(\overline{\operatorname{ch}(k)}),(\overline{\operatorname{ch}(k)})^{-1}\right] \text {. }
\end{aligned}
$$

The equation (24b) together with (181) show that $\tilde{\mathbf{W}}(\overline{\operatorname{ch}(2 k)})$ is in $L^{2}$ uniformly in $N$. Finally, the spectral theorem (as in (30) of [22]) is used to express $\tilde{\mathbf{W}}(\overline{\operatorname{ch}(k)})$ in terms of $\tilde{\mathbf{W}}(\overline{\operatorname{ch}(2 k)})$ we see that $\tilde{w}$ is a Hilbert-Schmidt operator uniformly in $N$. Its trace (97) is part of $X_{0}$, and

$$
\operatorname{Nor}\left(\mathcal{I}\left(\begin{array}{cc}
\tilde{w}^{T} & 0 \\
0 & -\tilde{w}
\end{array}\right)\right)
$$

is bounded on the first five slots of Fock space uniformly in $N$.

\section{REFERENCES}

[1] V. Bach, S. Breteaux, T. Chen, J. Fröhlich, I. M. Sigal: The time-dependent Hartree-Fock-Bogoliubov equation for Bosons. arXiv:1602.05171

[2] Ben Arous, G., Kirkpatrick, K., Schlein, B. : A central limit theorem in manybody quantum dynamics, Communications in Mathematical Physics, (321), (20130, pp. 371-417

[3] Benedikter, N., de Oliveira, G., Schlein B. : Quantitative Derivation of the Gross-Pitaevskii Equation, Communications on Pure and Applied Mathematics Volume 68, Issue 8, pages 1399 - 1482, 2015

[4] Boccato, C. , Cenatiempo, N., Schlein, B. : Quantum many-body fluctuations around nonlinear Schr odinger dynamics arXiv:1509.03837v1

[5] Bogoliubov, N. N.:On the theory of superfluidity, Journal of Physics, Vol XI, (1947)

[6] Chen, T. and Pavlović, N. : Derivation of the cubic NLS and Gross-Pitaevskii hierarchy for manybody dynamics in $d=2,3$ based on spacetime estimates, Ann. H. Poincare 15, No. 3 (2014), 543-588

[7] T. Chen, C. Hainzl, N. Pavlovic and R. Seiringer : Unconditional uniqueness for the cubic Gross-Pitaevskii hierarchy via quantum de Finetti, to appear in Commun. Pure Appl. Math.

[8] Chen, X. : Second order corrections to mean field evolution for weakly interacting bosons in the case of 3 body interactions, Arch. Rational Mech. Anal. 203 (2012), 455-497

[9] Chen, X. : On the Rigorous Derivation of the 3D Cubic Nonlinear Schrdinger Equation with A Quadratic Trap, Archive for Rational Mechanics and Analysis, 210 (2013), 365-408 
[10] Chen, X., Holmer, J., On the Klainerman-Machedon Conjecture of the Quantum BBGKY Hierarchy with Self-interaction, to appear in Journal of the European Mathematical Society.

[11] Chen, X., Holmer, J.: Correlation structures, Many-body Scattering Processes and the Deriva tion of the Gross-Pitaevskii Hierarchy, to appear in IMRN.

[12] Chen, X., Holmer, J.: Focusing Quantum Many-body Dynamics: The Rigorous Derivation of the 1D Focusing Cubic Nonlinear Schrdinger Equation , arXiv:1308.3895

[13] Erdös, L., Yau, H. T.: Derivation of the non-linear Schrödinger equation from a many-body Coulomb system. Adv. Theor. Math. Phys. 5, 1169-1205 (2001)

[14] Erdös, L., Schlein, B., Yau, H. T.: Derivation of the Gross-Pitaevskii hierarchy for the dynamics of Bose-Einstein condensate. Comm. Pure Appl. Math. 59, 1659-1741 (2006)

[15] Erdös, L., Schlein, B., Yau, H. T.: Derivation of the cubic non-linear Schrödinger equation from quantum dynamics of many-body systems. Invent. Math. 167, 515-614 (2007)

[16] Erdös, L., Schlein, B., Yau, H. T.: Rigorous derivation of the Gross-Pitaevskii equation. Phys. Rev. Lett. 98, 040404 (2007)

[17] Erdös, L., Schlein, B., Yau, H. T.: Derivation of the Gross-Pitaevskii equation for the dynamics of Bose-Einstein condensate. Annals Math. 172, 291-370 (2010)

[18] Erdös, L., Schlein, B., Yau, H. T.:The ground state energy of a low density Bose gas: a second order upper bound Physical Review A 78(5), (2008)

[19] Frölich, J., Knowles, A., Schwarz, S., : On the mean-field limit of Bosons with Coulomb two-body interaction, Comm. Math Phys., 288, (209), 1023-1059.

[20] Ginibre, J., Velo, G.: The classical field limit of scattering theory for nonrelativistic many-boson systems, I and II. Comm. Math. Phys. 66, 37-76 (1979) and 68, 45-68 (1979)

[21] Grillakis, M. Machedon. M, Margetis, D.: Second-order corrections to mean field evolution of weakly interacting Bosons. I. Comm. Math. Phys. 294, 273301 (2010)

[22] Grillakis, M. Machedon. M, Margetis, D.: Second-order corrections to mean field evolution of weakly interacting Bosons. II. Adv. in Math. 228, 1788-1815 (2011)

[23] Grillakis, M. Machedon. M,: Pair excitations and the mean field approximation of interacting Bosons, I. Communications in Mathematical Physics, Volume 324, Issue 2, pp 601-636, Oct (2013)

[24] Grillakis, M. Machedon. M, : Beyond mean field: On the role of pair excitations in the evolution of condensates, Journal of fixed point theory and applications, vol 14, no 1, (The Yvonne Choquet-Bruhat Festschrift), pp 91-111, December 2013.

[25] Hepp, K.: The classical limit for quantum mechanical correlation functions. Comm. Math. Phys. 35, 265-277 (1974)

[26] T. Kato and G. Ponce, Commutator estimates and the Euler and NavierStokes equations, Comm. Pure Appl. Math.41, 891907, (1988)

[27] Keel, M., Tao, T., Endpoint Strichartz estimates, Amer. J. Math., 120, 955980, (1998) 
[28] Knowles, A., Pickl, P. : Mean-field dynamics: Singular potentials and rate of convergence, Comm. Math Phys 298, 101-138 (2010).

[29] Kirkpatrick, K., Schlein, B., Staffilani, G. :Derivation of the two dimensional nonlinear Schrödinger equation from manybody quantum dinamics, Amer. J. Math, 133 (2011), 91-130.

[30] Klainerman, S., Machedon, M.: Space-time estimates for null forms and the local existence theorem. Comm. Pure Appl. Math. 46, 1221-1268 (1993)

[31] Klainerman, S., Machedon,M. On the uniqueness of solutions to the GrossPitaevskii hierarchy. Comm. Math. Phys. 279, 169-185 (2008)

[32] Kuz, E. Rate of convergence to mean field for interacting Bosons, Communications in PDE, 40:10, 1831-1854 (2015)

[33] Kuz, E. Exact evolution versus mean field with second order correction for Bosons interacting via short-range two-body potential, preprint

[34] Lee, T. D., Huang, K., Yang, C. N.: Eigenvalues and eigenfunctions of a Bose system of hard spheres and its low-temperature properties. Phys. Rev. 106, 1135-1145 (1957)

[35] Lewin, M. , P. T. Nam, and Schlein, B: Fluctuations around Hartree states in the mean-field regime. To appear in Amer. J. Math., 2015. (arXiv:1307.0665)

[36] Lieb, E. H., Seiringer: Proof of Bose-Einstein condensation for dilute trapped gasses, Phys. Rev. Letters 88 (2002), 170409.

[37] Lieb, E. H., Seiringer, R., Solovej, J. P., Yngvanson, J.: The mathematics of the Bose gas and its condensation. Basel, Switzerland: Birkhaüser Verlag, 2005

[38] Margetis, D.: Studies in classical electromagnetic radiation and Bose-Einstein condensation. Ph.D. thesis, Harvard University, 1999

[39] Margetis, D.: Solvable model for pair excitation in trapped Boson gas at zero temperature. J. Phys. A: Math. Theor. 41, 235004 (2008); Corrigendum. J. Phys. A: Math. Theor. 41, 459801 (2008)

[40] P. T. Nam, M. Napiorkowski : Bogoliubov correction to the mean-field dynamics of interacting Bosons, preprint.

[41] Rodnianski, I., Schlein, B.: Quantum fluctuations and rate of convergence towards mean field dynamics. Comm. Math. Phys. 291(2), 31-61 (2009)

[42] Shale, D.: Linear symmetries of free Boson fields, Trans. Amer. Math. Soc. 103(1), 149-167 (1962)

[43] T. Tao, Nonlinear dispersive equations: local and global analysis, CBMS regional series in mathematics, (2006)

[44] $\mathrm{Wu}, \mathrm{T}$. T.: Some nonequilibrium properties of a Bose system of hard spheres at extremely low temperatures. J. Math. Phys. 2, 105-123 (1961)

[45] H.-T. Yau and J. Yin, The second order upper bound for the ground energy of a Bose gas. J. Stat. Phys 136 453-503 (2009)

University of Maryland, College Park

E-mail address: mng@math.umd.edu

University of Maryland, College Park

E-mail address: mxm@math.umd.edu 